\title{
Basic Properties of Non-Stationary Ruijsenaars Functions
}

Edwin LANGMANN ${ }^{\dagger}$, Masatoshi NOUMI ${ }^{\ddagger}$ and Junichi SHIRAISHI ${ }^{\S}$

${ }^{\dagger}$ Physics Department, KTH Royal Institute of Technology, SE-106 91 Stockholm, Sweden

E-mail: langmann@kth.se

$\ddagger$ Department of Mathematics, KTH Royal Institute of Technology, SE-100 44 Stockholm, Sweden (on leave from: Department of Mathematics, Kobe University, Rokko, Kobe 657-8501, Japan)

E-mail: noumi@kth.se

$\S$ Graduate School of Mathematical Sciences, The University of Tokyo, Komaba, Tokyo 153-8914, Japan

E-mail: shiraish@ms.u-tokyo.ac.jp

Received June 15, 2020, in final form October 08, 2020; Published online October 21, 2020

https://doi.org/10.3842/SIGMA.2020.105

\begin{abstract}
For any variable number, a non-stationary Ruijsenaars function was recently introduced as a natural generalization of an explicitly known asymptotically free solution of the trigonometric Ruijsenaars model, and it was conjectured that this non-stationary Ruijsenaars function provides an explicit solution of the elliptic Ruijsenaars model. We present alternative series representations of the non-stationary Ruijsenaars functions, and we prove that these series converge. We also introduce novel difference operators called $\mathcal{T}$ which, as we prove in the trigonometric limit and conjecture in the general case, act diagonally on the non-stationary Ruijsenaars functions.
\end{abstract}

Key words: elliptic integrable systems; elliptic hypergeometric functions; Ruijsenaars systems

2020 Mathematics Subject Classification: 81Q80; 32A17; 33E20; 33E30

\section{Introduction}

The celebrated quantum Calogero-Moser-Sutherland systems [16] have natural relativistic generalizations discovered by Ruijsenaars [17]. The Ruijsenaars systems come in four kinds: rational, trigonometric, hyperbolic, and elliptic, with the latter case being the most general and reducing to the others in certain limits [17]. While the explicit solution of the trigonometric Ruijsenaars model is known since a long time: it is given by the celebrated Macdonald polynomials [13], and a construction of eigenfunctions of the hyperbolic model was completed recently [10], only partial results about the explicit solution in the general elliptic case exist $[7,18,19]$. Recently, one of us (S) conjectured an explicit solution of the elliptic Ruijsenaars model as a limit of special functions defined by explicit formal power series and called non-stationary Ruijsenaars functions [21]. In particular, it was shown in [21] that these functions reduce to the known solutions of the trigonometric Ruijsenaars model in the trigonometric limit; they have several remarkable symmetry properties; and they arise in a quantum field theory related to the elliptic Ruijsenaars system in a way that is a natural generalization of how the known solutions of the trigonometric Ruijsenaars model arise in a quantum field theory related to the trigonometric

This paper is a contribution to the Special Issue on Elliptic Integrable Systems, Special Functions and Quantum Field Theory. The full collection is available at https://www.emis.de/journals/SIGMA/elliptic-integrablesystems.html 
Ruijsenaars model (this is only a partial list of results in [21]). The validity of this conjecture was also tested by symbolic computer computations.

In this paper we prove some properties of the non-stationary Ruijsenaars functions which, we hope, will be useful to find proofs of the conjectures in [21]. In particular, we give alternative representations of these functions which are simpler than the original definitions; we prove that the series defining these functions are absolutely convergent in a suitable domain; and we present novel difference operators, called $\mathcal{T}$, which, we conjecture, acts diagonally on the non-stationary Ruijsenaars functions (by this we mean that the latter are eigenfunctions of the former).

Notation: Throughout the paper, the symbols $q, t, p, \kappa$ (complex parameters) and $N$ (variable number) have special significance. We use the following standard notation,

$$
\begin{aligned}
& (z ; q)_{\infty} \equiv \prod_{n=0}^{\infty}\left(1-z q^{n}\right) \quad(|q|<1), \\
& (z ; q)_{k} \equiv \frac{(z ; q)_{\infty}}{\left(q^{k} z ; q\right)_{\infty}} \quad(k \in \mathbb{Z}), \\
& (z ; q, p)_{\infty} \equiv \prod_{n, m=0}^{\infty}\left(1-q^{n} p^{m} z\right) \quad(|q|<1,|p|<1), \\
& \theta(z ; p) \equiv(z ; p)_{\infty}(p / z ; p)_{\infty}
\end{aligned}
$$

for $z \in \mathbb{C}$. Moreover, $T_{q, z}=q^{z \partial_{z}}$, i.e.,

$$
\left(T_{q, z} f\right)(z)=f(q z)
$$

for functions $f(z)$ of $z \in \mathbb{C}$. For $z \in \mathbb{C}, \operatorname{Re}(z)$ and $\operatorname{Im}(z)$ are the real- and imaginary parts of $z$, and $\sin \arg (z)=\operatorname{Im}(z) /|z|$. For $x=\left(x_{1}, \ldots, x_{N}\right)$ and $\lambda=\left(\lambda_{1}, \ldots, \lambda_{N}\right), x^{\lambda}$ is short for $x_{1}^{\lambda_{1}} \cdots x_{N}^{\lambda_{N}}, x^{-1}$ is short for $\left(x_{1}^{-1}, \ldots, x_{N}^{-1}\right)$, and $x^{+1}=x$. We denote as $\mathbb{C}\left[\left[z_{1}, \ldots, z_{N}\right]\right]$ the space of all formal power series $f(z)=\sum_{\mu \in \mathbb{Z}_{\geq 0}^{N}} c_{\mu} z_{1}^{\mu_{1}} \cdots z_{N}^{\mu}$ in formal variables $z=\left(z_{1}, \ldots, z_{N}\right)$ with complex coefficients $c_{\mu}$.

\section{Prerequisites}

We recall some known facts about the Macdonald polynomials [13] and certain special functions generalizing the Macdonald polynomials and constructed so as to solve the trigonometric Ruijsenaars model [15, 20] (Section 2.1). We also recall the eigenvalue problem defining the elliptic Ruijsenaars model, and the definition of the non-stationary Ruijsenaars functions (Section 2.2).

\subsection{Trigonometric Ruijsenaars model}

For fixed $N \in \mathbb{Z}_{\geq 1}$, the Macdonald polynomials $P_{\lambda}(x ; q, t)=P_{\lambda}\left(x ; q^{-1}, t^{-1}\right)$ are symmetric polynomials in variables $x=\left(x_{1}, \ldots, x_{N}\right) \in \mathbb{C}^{N}$ depending on two complex parameters $q$, $t$ and labeled by partitions $\lambda$ of length less than or equal to $N$, i.e., $\lambda=\left(\lambda_{1}, \ldots, \lambda_{N}\right)$ with $\lambda_{i} \in \mathbb{Z}_{\geq 0}$ such that $\lambda_{1} \geq \lambda_{2} \geq \cdots \geq \lambda_{N} \geq 0$. They can be defined as common eigenfunctions of the following commuting Macdonald-Ruijsenaars operators,

$$
D_{N}^{ \pm}(x \mid q, t) \equiv \sum_{i=1}^{N} \prod_{j \neq i}^{N} \frac{\left(1-t^{ \pm 1} x_{i} / x_{j}\right)}{\left(1-x_{i} / x_{j}\right)} T_{q, x_{i}}^{ \pm 1}
$$

with corresponding eigenvalues $\sum_{i=1}^{N} t^{ \pm(N-i)} q^{ \pm \lambda_{i}}$, together with a convenient normalization condition [13]. 
The operators $D_{N}^{ \pm}(x \mid q, t)$ are related by similarity transformations to the operators defining the trigonometric Ruijsenaars model [17].

As conjectured by one of us (S) [20] and proved by two of us (NS) [15], these eigenfunctions are naturally generalized to a special function $f_{N}(x|s| q, t)$ depending on another set of variables, $s=\left(s_{1}, \ldots, s_{N}\right) \in \mathbb{C}^{N}$, and determined by the following requirement, up to normalization: for $\lambda \in \mathbb{C}^{N}$, the function

$$
x^{\lambda} f_{N}(x|s| q, t), \quad s_{i}=t^{N-i} q^{\lambda_{i}}
$$

is a common eigenfunction of $D_{N}^{ \pm}(x \mid q, t)$ with corresponding eigenvalue $\sum_{j=1}^{N} s_{j}^{ \pm 1}$; if $\lambda$ is a partition, then the function in (2.2) is equal to the Macdonald polynomial $P_{\lambda}(x ; q, t)$ [15]. The function $f_{N}(x|s| q, t)$ is called the asymptotically free solution of the trigonometric Ruijsenaars model.

One remarkable property of this function is that it has a simple explicit series representation which converges absolutely in a suitable domain $[15]:^{1}$

$$
f_{N}(x|s| q, t)=\sum_{\theta \in \mathrm{M}_{N}} c_{N}(\theta|s| q, t) \prod_{1 \leq i<k \leq N}\left(x_{k} / x_{i}\right)^{\theta_{i k}}
$$

with $\mathrm{M}_{N}$ the set of $N \times N$ strictly upper triangular matrices with nonnegative integer entries:

$$
\mathrm{M}_{N} \equiv\left\{\theta=\left(\theta_{i k}\right)_{i, k=1}^{N} \mid \theta_{i k} \in \mathbb{Z}_{\geq 0}(\forall i, k), \theta_{i k}=0(1 \leq k \leq i \leq N)\right\},
$$

and $^{2}$

$$
\begin{aligned}
c_{N}(\theta|s| q, t)= & \prod_{i=1}^{N} \prod_{i<j \leq k \leq N} \frac{\left(q^{\sum_{a>k}\left(\theta_{i a}-\theta_{j a}\right)} t s_{j} / s_{i} ; q\right)_{\theta_{i k}}}{\left(q^{\sum_{a>k}\left(\theta_{i a}-\theta_{j a}\right)} q s_{j} / s_{i} ; q\right)_{\theta_{i k}}} \\
& \times \prod_{i=1}^{N} \prod_{i \leq j<k \leq N} \frac{\left(q^{-\theta_{j k}-\sum_{a>k}\left(\theta_{j a}-\theta_{i a}\right)} q s_{j} / t s_{i} ; q\right)_{\theta_{i k}}}{\left(q^{-\theta_{j k}-\sum_{a>k}\left(\theta_{j a}-\theta_{i a}\right)} s_{j} / s_{i} ; q\right)_{\theta_{i k}}}
\end{aligned}
$$

(note that (2.3)-(2.5) is equivalent to (1.10)-(1.11) in [15]).

For later reference, we also define the function ${ }^{3}$

$$
\varphi_{N}(x|s| q, t) \equiv \prod_{1 \leq i<j \leq N} \frac{\left(q x_{j} / t x_{i} ; q\right)_{\infty}}{\left(q x_{j} / x_{i} ; q\right)_{\infty}} f_{N}(x|s| q, t),
$$

which, as proved in [15], has the following remarkably symmetry properties:

$$
\begin{aligned}
& \varphi_{N}(x|s| q, t)=\varphi_{N}(s|x| q, t) \quad \text { (bispectral duality), } \\
& \varphi_{N}(x|s| q, t)=\varphi_{N}(s|x| q, q / t) \quad \text { (Poincaré duality). }
\end{aligned}
$$

\subsection{Non-stationary Ruijsenaars functions}

The analogue of the operators in (2.1) for the elliptic Ruijsenaars model depends on a further complex parameter, $p$ such that $|p|<1$ :

$$
D_{N}^{ \pm}(x \mid q, t, p) \equiv \sum_{i=1}^{N} \prod_{j \neq i}^{N} \frac{\theta\left(t^{ \pm 1} x_{i} / x_{j} ; p\right)}{\theta\left(x_{i} / x_{j} ; p\right)} T_{q, x_{i}}^{ \pm 1}
$$

with the theta function $\theta(z ; p)$ given in the introduction; note that $D_{N}^{ \pm}(x \mid q, t)=D_{N}^{ \pm}(x \mid q, t, 0)$.

\footnotetext{
${ }^{1}$ Note that $f_{N}(x|s| q, t)$ here is $p_{N}(x ; s \mid q, t)$ in [15].

${ }^{2}$ We write (2.5) in a way that emphasizes the similarity with (3.3) below, for reasons that will become clear later on. Due to this, we include the empty factors for $i=N$.

${ }^{3}$ Note that $\varphi_{N}(x|s| q, t)$ here is $\psi_{N}(x ; s \mid q, t)$ in [15].
} 
The non-stationary Ruijsenaars function $f^{\widehat{\mathfrak{g l}}_{N}}(x, p|s, \kappa| q, t)$ is a conjectured eigenfunction of a deformation of the operators in (2.8), depending on a further complex parameter, $\kappa$, and reducing to the operators in (2.8) in the limit $\kappa \rightarrow 1$ [21].

Definition 2.1 (non-stationary Ruijsenaars functions). For $N \in \mathbb{Z}_{\geq 1}$, four parameters $q, t, p, \kappa$, and two sets of variables $x=\left(x_{1}, \ldots, x_{N}\right)$ and $s=\left(s_{1}, \ldots, s_{N}\right)$, the non-stationary Ruijsenaars function is defined as a formal power series in $\left(p x_{2} / x_{1}, \ldots, p x_{N} / x_{N-1}, p x_{1} / x_{N}\right)$ as follows,

$$
f^{\widehat{\mathfrak{g l}}_{N}}(x, p|s, \kappa| q, t) \equiv \sum_{\lambda^{(1)}, \ldots, \lambda^{(N)} \in \mathrm{P}} \prod_{i, j=1}^{N} \frac{\mathrm{N}_{\lambda^{(i)}, \lambda^{(j)}}^{(j-i \mid N)}\left(t s_{j} / s_{i} \mid q, \kappa\right)}{\mathrm{N}_{\lambda^{(i)}, \lambda^{(j)}}^{(j-i \mid N)}\left(s_{j} / s_{i} \mid q, \kappa\right)} \prod_{\beta=1}^{N} \prod_{\alpha \geq 1}\left(p x_{\alpha+\beta} / t x_{\alpha+\beta-1}\right)^{\lambda_{\alpha}^{(\beta)}}
$$

with $x_{\alpha+\ell N} \equiv x_{\alpha}$ for all $\alpha=1, \ldots, N$ and $\ell \in \mathbb{Z}_{\geq 1}, \mathrm{P}$ the set of all partitions $\lambda$ of arbitrary length, i.e., $\lambda=\left(\lambda_{1}, \lambda_{2}, \ldots\right)$ with $\lambda_{i} \in \mathbb{Z}_{\geq 0}$ such that $\lambda_{1} \geq \lambda_{2} \geq \cdots$ and $\lambda_{i}=0$ for $i \gg 0$, and

$$
\mathrm{N}_{\lambda, \mu}^{(k \mid N)}(u \mid q, \kappa) \equiv \prod_{\substack{b \geq a \geq 1 \\ b-a \equiv k(\bmod N)}}\left(u q^{-\mu_{a}+\lambda_{b+1}} \kappa^{-a+b} ; q\right)_{\substack{\lambda_{b}-\lambda_{b+1} \\ \beta-\alpha \equiv(-\bar{k}-\overline{1})(\bmod N)}}\left(u q^{\lambda_{\alpha}-\mu_{\beta}} \kappa^{\alpha-\beta-1} ; q\right)_{\mu_{\beta}-\mu_{\beta+1}}
$$

for $\lambda, \mu \in \mathrm{P}, k \in \mathbb{Z} / N \mathbb{Z}$, and $u \in \mathbb{C}$.

As discussed in [21], the expressions in (2.10) are Nekrasov factors [14]. Moreover, by changing $(p, \kappa) \rightarrow\left(p^{1 / N}, \kappa^{1 / N}\right)$ and scaling variables $x \rightarrow p^{\delta / N} x=\left(p^{(N-1) / N} x_{1}, p^{(N-2) / 2} x_{2}, \ldots, x_{N}\right)$ and similarly for $s$, one obtains a function, $f^{\widehat{\mathfrak{g l}}_{N}}\left(p^{\delta / N} x, p^{1 / N}\left|\kappa^{\delta / N} s, \kappa^{1 / N}\right| q, q / t\right)$, that converges to the asymptotically free solution of the Ruijsenaars model, $f_{N}(x|s| q, t)$, in the limit $p \rightarrow 0$ [21].

Remark 2.2. To explain the scaling just mentioned, we point out one important technical point: in Definition 2.1, equations (2.11) and (2.12) below, and equations (4.10) and (4.11) in Section 4 , we use balanced coordinates $x_{B}, p_{B}, s_{B}, \kappa_{B}$ and $t_{B}$ (written without the subscript $B$ for simplicity), whereas elsewhere in the paper we use unbalanced coordinates $x_{U}, p_{U}, s_{U}, \kappa_{U}$ and $t_{U}$ (also written without subscript $U$ ) related to the balanced coordinates as follows,

$$
\begin{array}{lll}
\left(x_{B}\right)_{i}=\left(p_{U}\right)^{(N-i) / N}\left(x_{U}\right)_{i} & (i=1, \ldots, N), & p_{B}=\left(p_{U}\right)^{N}, \\
\left(s_{B}\right)_{i}=\left(\kappa_{U}\right)^{(N-i) / N}\left(s_{U}\right)_{i} & (i=1, \ldots, N), & \kappa_{B}=\left(\kappa_{U}\right)^{N}, \quad t_{B}=q / t_{U} .
\end{array}
$$

Thus, the scaling just described can be understood as a transformation from balanced to unbalanced coordinates.

The main conjecture in [21] is that eigenfunction of the operator in (2.8) can be obtained by dividing this rescaled function $f^{\widehat{\mathfrak{g l}_{N}}}\left(p^{\delta / N} x, p^{1 / N}\left|\kappa^{\delta / N} s, \kappa^{1 / N}\right| q, q / t\right)$ by a (known) factor $\alpha\left(p^{1 / N}\left|\kappa^{\delta / N} s, \kappa^{1 / N}\right| q, t\right)$ and taking the limit $\kappa \rightarrow 1$; see Conjecture 1.14 in [21]. One important open problem is to find the operator depending on $\kappa$ having these rescaled non-stationary Ruijsenaars functions as eigenfunctions and reducing to the Macdonald-Ruijsenaars operator in (2.8) in the limit $\kappa \rightarrow 1 .{ }^{4}$ At this point, this operator is only known in limiting cases: the non-relativistic limit $q \rightarrow 1$ where the Ruijsenaars systems reduce to the non-stationary elliptic Calogero-Sutherland system [21], and the limit $t, p \rightarrow 0$ with fixed $p / t$ leading to the affine Toda system [21]. We stress that the non-stationary $\mathcal{T}$-operators introduced in this paper do not reduce to the elliptic Macdonald-Ruijsenaars operators in the limit $\kappa \rightarrow 1$ : the $\mathcal{T}$-operators are of a different kind, and they are new even in the trigonometric limit; only the affine Toda limit of the non-stationary $\mathcal{T}$-operator was known before [21].

\footnotetext{
${ }^{4}$ There is, however, a recent proposal mentioned in Section 5.
} 
A natural generalization of the function in (2.6) is

$$
\begin{aligned}
\varphi^{\widehat{\mathfrak{g l}}_{N}}(x, p|s, \kappa| q, t) \equiv & \prod_{1 \leq i<j \leq N} \frac{\left(q p^{j-i} x_{j} / t x_{i} ; q, p^{N}\right)_{\infty}}{\left(q p^{j-i} x_{j} / x_{i} ; q, p^{N}\right)_{\infty}} \\
& \times \prod_{1 \leq i \leq j \leq N} \frac{\left(q p^{N-j+i} x_{i} / t x_{j} ; q, p^{N}\right)_{\infty}}{\left(q p^{N-j+i} x_{i} / x_{j} ; q, p^{N}\right)_{\infty}} f^{\mathfrak{g} l_{N}}(x, p|s, \kappa| q, t)
\end{aligned}
$$

and, as conjectured in [21], it has the following symmetry properties generalizing the ones in $(2.7)$.

Conjecture 2.3. The functions in (2.11) satisfy

$$
\begin{aligned}
& \varphi^{\widehat{\mathfrak{g}}_{N}}(x, p|s, \kappa| q, t)=\varphi^{\widehat{\mathfrak{g} l}} \widehat{\Upsilon}_{N}(s, \kappa|x, p| q, t) \quad \text { (bispectral duality) }, \\
& \varphi^{\widehat{\mathfrak{g l}}_{N}}(x, p|s, \kappa| q, t)=\varphi^{\widehat{\mathfrak{g l}}_{N}}(x, p|s, \kappa| q, q / t) \quad \text { (Poincaré duality). }
\end{aligned}
$$

\section{Results on the non-stationary Ruijsenaars function}

We give alternative series representations of the non-stationary Ruijsenaars functions (Section 3.1) and prove convergence of these series in a suitable domain (Section 3.2).

\subsection{Alternative series representations}

Our first result makes manifest that the non-stationary Ruijsenaars function in (2.9)-(2.10) is a natural generalization of the asymptotically free solutions of the trigonometric Ruijsenaars model in (2.3)-(2.5). For that, we extend the variables $x=\left(x_{i}\right)_{i=1}^{N}$ and $s=\left(s_{i}\right)_{i=1}^{N}$ to infinitely many variables $\bar{x}=\left(x_{i}\right)_{i=1}^{\infty}$ and $\bar{s}=\left(s_{i}\right)_{i=1}^{\infty}$; as we will see, the pertinent extension is provided by the parameters $p$ and $\kappa$, respectively - see (3.5).

We first introduce a natural generalization of the function in (2.3)-(2.5) to infinitely many variables.

Definition 3.1. For $N \in \mathbb{Z}_{\geq 1}$, two parameters $q, t$, and two sets of infinitely many variables $\bar{x}=\left(x_{1}, x_{2}, \ldots\right)$ and $\bar{s}=\left(s_{1}, s_{2}, \ldots\right)$, let the following define a formal power series in the infinitely many variables $\left(x_{2} / x_{1}, x_{3} / x_{2}, x_{4} / x_{3}, \ldots\right)$,

$$
f_{N, \infty}(\bar{x}|\bar{s}| q, t) \equiv \sum_{\theta \in \hat{\mathrm{M}}_{N}} c_{N, \infty}(\theta|\bar{s}| q, t) \prod_{i=1}^{N} \prod_{k>i}\left(x_{k} / x_{i}\right)^{\theta_{i k}}
$$

with $\hat{\mathrm{M}}_{N}$ the set of infinite, $N$-periodic, strictly upper triangular matrices with nonnegative integer entries which are non-zero only in a finite strip away from the diagonal:

$$
\hat{\mathrm{M}}_{N} \equiv\left\{\theta=\left(\theta_{i k}\right)_{i, k=1}^{\infty} \mid \theta_{i k}=\theta_{i+N, k+N} \in \mathbb{Z}_{\geq 0}(i, k \geq 1), \theta_{i k}=0(k \leq i, k \gg i)\right\},
$$

and

$$
\begin{aligned}
c_{N, \infty}(\theta|\bar{s}| q, t) \equiv & \prod_{i=1}^{N} \prod_{i<j \leq k<\infty} \frac{\left(q^{\sum_{a>k}\left(\theta_{i a}-\theta_{j a}\right)} t s_{j} / s_{i} ; q\right)_{\theta_{i k}}}{\left(q^{\sum_{a>k}\left(\theta_{i a}-\theta_{j a}\right)} q s_{j} / s_{i} ; q\right)_{\theta_{i k}}} \\
& \times \prod_{i=1}^{N} \prod_{i \leq j<k<\infty} \frac{\left(q^{-\theta_{j k}-\sum_{a>k}\left(\theta_{j a}-\theta_{i a}\right)} q s_{j} / t s_{i} ; q\right)_{\theta_{i k}}}{\left(q^{-\theta_{j k}-\sum_{a>k}\left(\theta_{j b}-\theta_{i a}\right)} s_{j} / s_{i} ; q\right)_{\theta_{i k}}} .
\end{aligned}
$$


Note that the product in (3.3) always contains only a finite number of factors different from 1. Moreover, by the condition $\theta_{i k}=\theta_{i+N, k+N}$, a matrix $\theta \in \hat{\mathrm{M}}_{N}$ is fully determined by the matrix elements $\theta_{i k}$ for $1 \leq i \leq N$ and $1 \leq k<\infty$. Furthermore, matrices in $\mathrm{M}_{N}$ can be naturally identified with matrices $\theta$ in $\hat{\mathrm{M}}_{N}$ by setting $\theta_{i k}=0$ if $i>N$, or $k>N$, or both.

To state out result we use the $N$-vector $\delta \equiv\left(\delta_{1}, \ldots, \delta_{N}\right)$ with $\delta_{i}=N-i$, and the notation $p^{\delta / N} x$ and $\kappa^{\delta / N} s$ for the $N$-vectors with components $\left(p^{\delta / N} x\right)_{i}=p^{(N-i) / N} x_{i}$ and $\left(\kappa^{\delta / N} s\right)_{i}=$ $\kappa^{(N-i) / N} s_{i}$, respectively $(i=1, \ldots, N)$. As explained in Remark 2.2, this can be understood as a transformation going from balanced to unbalanced coordinates.

Theorem 3.2. The non-stationary Ruijsenaars function in (2.9)-(2.10) is related to the function in (3.1)-(3.3) as follows,

$$
f^{\widehat{\mathfrak{g l}}_{N}}\left(p^{\delta / N} x, p^{1 / N}\left|\kappa^{\delta / N} s, \kappa^{1 / N}\right| q, q / t\right)=f_{N, \infty}(\bar{x}|\bar{s}| q, t)
$$

with the variables $x=\left(x_{i}\right)_{i=1}^{N}$ and $s=\left(s_{i}\right)_{i=1}^{N}$ on the left-hand side extended to variables $\bar{x}=$ $\left(x_{i}\right)_{i=1}^{\infty}$ and $\bar{s}=\left(s_{i}\right)_{i=1}^{\infty}$ on the right-hand side by the rules ${ }^{5}$

$$
x_{i+N}=p x_{i}, \quad s_{i+N}=\kappa s_{i} \quad(i \geq 1) .
$$

(The proof is by straightforward computations given in Appendix A.)

In the following, it is sometimes convenient to use a notation for the functions $f_{N, \infty}$ that emphasizes that the arguments $\bar{x}$ and $\bar{s}$ are fixed by $x, s, p$ and $\kappa$ :

Definition 3.3. We write

$$
f_{N, \infty}(x, p|s, \kappa| q, t) \equiv f_{N, \infty}(\bar{x}|\bar{s}| q, t)
$$

if $\bar{x}=\left(x_{1}, x_{2}, \ldots\right)$ and $\bar{s}=\left(s_{1}, s_{2}, \ldots\right)$ on the right-hand side are determined by $x=\left(x_{1}, \ldots, x_{N}\right)$, $p, s=\left(s_{1}, \ldots, s_{N}\right)$, and $\kappa$ as in (3.5). Thus

$$
f_{N, \infty}(x, p|s, \kappa| q, t)=\sum_{\theta \in \hat{\mathrm{M}}_{N}} c_{N, \infty}(\theta|s, \kappa| q, t) e_{N, \infty}(\theta \mid x, p)
$$

with

$$
c_{N, \infty}(\theta|s, \kappa| q, t) \equiv c_{N, \infty}(\theta|\bar{s}| q, t), \quad e_{N, \infty}(\theta \mid x, p) \equiv \prod_{i=1}^{N} \prod_{k=i+1}^{\infty}\left(x_{k} / x_{i}\right)^{\theta_{i k}}
$$

and the identifications in (3.5) on the right-hand side in (3.6).

Theorem 3.2 makes manifest the following important result in [21]: After suitably scaling the variables, the non-stationary Ruijsenaars function reduces the asymptotically free solution of the trigonometric Ruijsenaars model, $f_{N}(x|s| q, t)(2.3)-(2.5)$, in the limit $p \rightarrow 0$; in particular, it becomes independent of $\kappa$ in this limit:

Corollary 3.4. We have

$$
\lim _{p \rightarrow 0} f^{\widehat{\mathfrak{g l}}_{N}}\left(p^{\delta / N} x, p^{1 / N}\left|\kappa^{\delta / N} s, \kappa^{1 / N}\right| q, q / t\right)=f_{N}(x|s| q, t) .
$$

Proof. By Theorem 3.2, (3.7) is equivalent to

$$
\lim _{p \rightarrow 0} f_{N, \infty}(x, p|s, \kappa| q, t)=f_{N}(x|s| q, t),
$$

but this is obvious from definitions: by (3.5), $\left(x_{k} / x_{i}\right) \rightarrow 0$ for $k>N$ as $p \rightarrow 0$; therefore, the sum over $\theta \in \hat{\mathrm{M}}_{N}$ on the right-hand side in (3.1) collapses to a sum over $\theta \in \mathrm{M}_{N}$ in this limit; obviously, for $\theta \in \mathrm{M}_{N}$, the coefficients $c_{N, \infty}(\theta|\bar{s}| q, t)$ in (3.3) do not depend on $s_{i>N}$ and are identical with the coefficients $c_{N}(\theta|s| q, t)$ in (2.5).

\footnotetext{
5 " $x_{i+N}=p x_{i}(i \geq 1)$ " is short for " $x_{i+k N}=p^{k} x_{i}\left(i=1, \ldots, N, k \in \mathbb{Z}_{\geq 1}\right)$ ".
} 
We prove Theorem 3.2 by a direct computation in Appendix A. This proof uses an alternative representation of the function $f_{N, \infty}(\bar{x}|\bar{s}| q, t)$ which is interesting in its own right:

Lemma 3.5. The formal power series in (3.1)-(3.3) can be written as

$$
f_{N}(\bar{x}|\bar{s}| q, t)=\sum_{\boldsymbol{\lambda} \in \mathrm{P}^{N}} C_{N, \infty}(\boldsymbol{\lambda}|\bar{s}| q, t) \prod_{i=1}^{N} \prod_{k \geq 1}\left(x_{i+k} / x_{i+k-1}\right)^{\lambda_{k}^{(i)}},
$$

with $\mathrm{P}^{N}$ the set of all $N$-partitions $\boldsymbol{\lambda}=\left(\lambda^{(1)}, \lambda^{(2)}, \ldots, \lambda^{(N)}\right), \lambda^{(i)}$ a partition of arbitrary length for $i=1, \ldots, N$, and

$$
\begin{aligned}
C_{N, \infty}(\boldsymbol{\lambda}|\bar{s}| q, t)= & \prod_{i=1}^{N} \prod_{i<j \leq k<\infty} \frac{\left(q^{\lambda_{k-i+1}^{(i)}-\lambda_{k-j+1}^{(j)}} t s_{j} / s_{i} ; q\right)_{\lambda_{k-i}^{(i)}-\lambda_{k-i+1}^{(i)}}}{\left(q^{\lambda_{k-i+1}^{(i)}-\lambda_{k-j+1}^{(j)}} q s_{j} / s_{i} ; q\right)_{\lambda_{k-i}^{(i)}-\lambda_{k-i+1}^{(i)}}} \\
& \times \prod_{i=1}^{N} \prod_{i \leq j<k<\infty} \frac{\left(q^{-\lambda_{k-j}^{(j)}+\lambda_{k-i+1}^{(i)}} q s_{j} / t s_{i} ; q\right)_{\lambda_{k-i}^{(i)}-\lambda_{k-i+1}^{(i)}}}{\left(q^{-\lambda_{k-j}^{(j)}+\lambda_{k-i+1}^{(i)}} s_{j} / s_{i} ; q\right)_{\lambda_{k-i}^{(i)}-\lambda_{k-i+1}^{(i)}}}
\end{aligned}
$$

setting $\lambda_{j}^{(i+N)} \equiv \lambda_{j}^{(i)}$.

Proof. Straightforward computations, using that $\theta_{i k}=\lambda_{k-i}^{(i)}-\lambda_{k-i+1}^{(i)}$ defines a one-to-one correspondence between multi-partitions $\boldsymbol{\lambda}=\left(\lambda^{(1)}, \ldots, \lambda^{(N)}\right)$ in $\mathrm{P}^{N}$ and matrices $\theta=\left(\theta_{i k}\right)_{i, k=1}^{\infty}$ in $\hat{\mathrm{M}}_{N}$ (the interested reader can find the details in Appendix A.1).

It is interesting to note that

$$
\varphi_{N, \infty}(\bar{x}|\bar{s}| q, t) \equiv \prod_{i=1}^{N} \prod_{j>i} \frac{\left(q x_{j} / t x_{i} ; q\right)_{\infty}}{\left(q x_{j} / x_{i} ; q\right)_{\infty}} f_{N, \infty}(\bar{x}|\bar{s}| q, t)
$$

is a natural generalization of the function in (2.6) due to the following implication of Theorem 3.2.

Fact 3.6. The following holds,

$$
\varphi^{\widehat{\mathfrak{g l}}_{N}}\left(p^{\delta / N} x, p^{1 / N}\left|\kappa^{\delta / N} s, \kappa^{1 / N}\right| q, q / t\right)=\varphi_{N, \infty}(\bar{x}|\bar{s}| q, t)
$$

with the variables $x=\left(x_{i}\right)_{i=1}^{N}$ and $s=\left(x_{i}\right)_{i=1}^{N}$ on the left-hand side extended to variables $\bar{x}=\left(x_{i}\right)_{i=1}^{\infty}$ and $\bar{s}=\left(s_{i}\right)_{i=1}^{\infty}$ on the right-hand side by the rules in (3.5). Moreover, the conjectures in (2.12) are equivalent to

$$
\begin{aligned}
& \varphi_{N, \infty}(\bar{x}|\bar{s}| q, t)=\varphi_{N, \infty}(\bar{s}|\bar{x}| q, t) \quad \text { (bispectral duality) } \\
& \varphi_{N, \infty}(\bar{x}|\bar{s}| q, t)=\varphi_{N, \infty}(\bar{x}|\bar{s}| q, q / t) \quad \text { (Poincaré duality), }
\end{aligned}
$$

under the conditions in (3.5).

Proof. Since $p^{(j-i) / N}\left(p^{\delta / N} x\right)_{j} /\left(p^{\delta / N} x\right)_{i}=x_{j} / x_{i}$ for all $i, j=1, \ldots, N$, we only need to show that

$$
\prod_{i=1}^{N} \prod_{j>i} \frac{\left(q x_{j} / t x_{i} ; q\right)_{\infty}}{\left(q x_{j} / x_{i} ; q\right)_{\infty}}=\prod_{1 \leq i<j \leq N} \frac{\left(q x_{j} / t x_{i} ; q, p\right)_{\infty}}{\left(q x_{j} / x_{i} ; q, p\right)_{\infty}} \prod_{1 \leq i \leq j \leq N} \frac{\left(q p x_{i} / t x_{j} ; q, p\right)_{\infty}}{\left(q p x_{i} / x_{j} ; q, p\right)_{\infty}} .
$$

This is proved in Appendix D, Lemma D.1. 


\subsection{Convergence}

We prove that the non-stationary Ruijsenaars functions $f_{N}(x, p|s, \kappa| q, t)$ in Definitions 3.1 and 3.3 are absolutely convergent in a certain domain of variables and parameters.

Theorem 3.7. For fixed $N \in \mathbb{Z}_{\geq 1}$, assume that the variables $s=\left(s_{1}, \ldots, s_{N}\right) \in \mathbb{C}^{N}$ and the parameters $q$ and $\kappa$ satisfy the following conditions,

(i) for some $\sigma>0$,

$$
\left|\sin \arg \left(s_{i} / s_{j}\right)\right|>\sigma \quad(1 \leq i<j \leq N)
$$

(ii) $q$ and $\kappa$ both are real, and either $|q|<1$ and $|\kappa|>1$, or $|q|>1$ and $|\kappa|<1$.

Then, there exists a constant $\rho>0$ such that the formal power series

$$
f_{N, \infty}(x, p ; s, \kappa \mid q, t) \in \mathbb{C}\left[\left[x_{2} / x_{1}, \ldots, x_{N} / x_{N-1}, p x_{1} / x_{N}\right]\right]
$$

in Definitions 3.1 and 3.3 is absolutely convergent in the domain

$$
|p|<\rho^{N}, \quad\left|x_{2} / x_{1}\right|<\rho, \quad \ldots, \quad\left|x_{N} / x_{N-1}\right|<\rho, \quad\left|p x_{1} / x_{N}\right|<\rho .
$$

Remark 3.8. In our proof, we actually show convergence for any $\rho<1 / C_{1} C_{2}$ where

$$
\begin{aligned}
& C_{1}=1+|1-t / q| \max \left(\frac{1}{\sigma}, \frac{|\kappa|}{|1-| \kappa||}\right), \\
& C_{2}=1+|1-q / t| \max \left(\frac{1}{\sigma}, \frac{1}{|1-| q||}\right) .
\end{aligned}
$$

Remark 3.9. We believe that it is possible to refine this convergence result. In particular, we believe that there are regions of convergence where $s_{i} / s_{j}, 1 \leq i<j \leq N$, are real and $q$ and $\kappa$ have non-trivial imaginary parts.

Proof of Theorem 3.7. Our strategy of proof is to show that our assumptions imply simple upper bounds on the terms appearing in the series in (3.8)-(3.9):

$$
\left|\prod_{i=1}^{N} \prod_{k \geq 1}\left(x_{i+k} / x_{i+k-1}\right)^{\lambda_{k}^{(i)}}\right| \leq \rho^{|\boldsymbol{\lambda}|}, \quad\left|C_{N, \infty}(\boldsymbol{\lambda}|\bar{s}| q, t)\right| \leq C_{1}^{|\boldsymbol{\lambda}|} C_{2}^{|\boldsymbol{\lambda}|}
$$

with $|\boldsymbol{\lambda}| \equiv \sum_{i=1}^{N} \sum_{k \geq 1} \lambda_{k}^{(i)}$ and $\alpha=\rho C_{1} C_{2}<1$. With that, absolute convergence follows from the comparison test: the series in (3.8)-(3.9) is of the form $\sum_{\boldsymbol{\lambda} \in \mathrm{P}^{N}} a_{\boldsymbol{\lambda}}$ with $\left|a_{\boldsymbol{\lambda}}\right| \leq \alpha^{|\boldsymbol{\lambda}|}$ for all $\boldsymbol{\lambda} \in \mathrm{P}^{N}$, and the series $\sum_{\boldsymbol{\lambda} \in \mathrm{P}^{N}} \alpha^{|\boldsymbol{\lambda}|}$ converges absolutely for $|\alpha|<1$.

The first estimate in (3.13) is a simple consequence of the conditions in (3.11): since $x_{i+N}=p x_{i}$ for all $i \geq 1$, these conditions are equivalent to

$$
\left|x_{i+1} / x_{i}\right|<\rho \quad(i \geq 1),
$$

which clearly implies the result.

The proof of the second estimate in (3.13) is more involved and, for this reason, we supplement our somewhat descriptive arguments in the main text below by a detailed argument in Appendix B.

We observe that $C_{N, \infty}(\boldsymbol{\lambda}|\bar{s}| q, t)$ in (3.9) is a product of fractions $\left(1-q^{l} a u\right) /\left(1-q^{l} u\right)$ with $a=t / q$ in the first group of products and $a=q / t$ in the second group, $l \in \mathbb{Z}$, and $u=s_{j} / s_{i}$ 
for $i=1, \ldots, N$ and $j \geq i$; moreover, $s_{j+\ell N}=\kappa^{\ell} s_{j}$ for $\ell \in \mathbb{Z}_{\geq 1}$. Such a fraction can be estimated in a simple way:

$$
\left|\frac{1-q^{l} a u}{1-q^{l} u}\right|=\left|1+(1-a) \frac{q^{l} u}{1-q^{l} u}\right| \leq 1+|1-a|\left|\frac{q^{l} u}{1-q^{l} u}\right| .
$$

If $j-i$ is not an integer multiple of $N$, we can estimate this further using

$$
\left|\frac{z}{1-z}\right| \leq \frac{1}{|\sin \arg (z)|} \quad(z \in \mathbb{C} \backslash\{\mathbb{R}\})
$$

(to see that the latter inequality holds, write $z=|z| \mathrm{e}^{\mathrm{i} \varphi}$ and note that (3.14) is equivalent to

$$
|z|^{2} \sin ^{2} \varphi \leq 1+|z|^{2}-2|z| \cos \varphi \Leftrightarrow 0 \leq(1-|z| \cos \varphi)^{2}
$$

which is obvious). Since we assume that $q$ and $\kappa$ both are real,

$$
\left|\sin \arg \left(q^{l} \kappa^{\ell} s_{j} / s_{i}\right)\right|=\left|\sin \arg \left(s_{j} / s_{i}\right)\right| \geq \sigma>0 \quad\left(j-i \neq N \mathbb{Z}_{\geq 0}\right)
$$

for all integers $l, \ell$, we get a simple universal bound for these fractions:

$$
\left|\frac{1-q^{l} a s_{j} / s_{i}}{1-q^{l} s_{j} / s_{i}}\right| \leq 1+|1-a| \frac{1}{\sigma} \quad\left(j-i \notin N \mathbb{Z}_{\geq 0}\right)
$$

for all integers $l$. However, this bound does not work for $j=i+\ell N$ with $\ell \in \mathbb{Z}_{>0}$ since, in these cases, $q^{l} u=q^{l} s_{j} / s_{i}=q^{l} \kappa^{\ell}$ is real. However, one can check that, in all these latter cases, either $l \leq 0$ and $\ell>0$, or $l<0$ and $\ell \geq 0$, and thus, by our assumptions, $z \equiv q^{l} u=q^{l} \kappa^{\ell}$ always satisfies either $|z| \geq \min \left(|q|^{-1},|\kappa|\right)>1$ (if $|q|<1$ and $|\kappa|>1$ ) or $|z| \leq \max \left(|q|,|\kappa|^{-1}\right)<1$ (if $|q|>1$ and $|\kappa|<1$ ); we therefore can use the inequality

$$
\left|\frac{z}{1-z}\right| \leq \frac{|z|}{|1-| z||} \quad(|z| \neq 1)
$$

to get simple universal bounds for the cases $j=i+\ell N$ with $\ell \in \mathbb{Z}_{\geq 0}$ as well (we spell our the details of this argument in Appendix B.2.2). We thus get estimates

$$
\left|\frac{1-q^{l} a u}{1-q^{l} u}\right| \leq C_{1,2}
$$

with different upper bounds, $C_{1}$ and $C_{2}$, for all fractions in the first and second groups of products on the right-hand side in (3.9), respectively. The arguments above allow to compute the constants $C_{1}$ and $C_{2}$ and give the results in (3.12); the interested reader can find the details of this computation in Appendix B.

Inserting these bounds into (3.9) we obtain

$$
\begin{aligned}
\left|C_{N, \infty}(\boldsymbol{\lambda}|\bar{s}| q, t)\right| & \leq \prod_{i=1}^{N}\left(\prod_{i<j \leq k<\infty} C_{1}^{\lambda_{k-i}^{(i)}-\lambda_{k-i+1}^{(i)}}\right)\left(\prod_{i \leq j<k<\infty} C_{2}^{\lambda_{k-i}^{(i)}-\lambda_{k-i+1}^{(i)}}\right) \\
& =\prod_{i=1}^{N}\left(\prod_{i<j<\infty} C_{1}^{\lambda_{j-i}^{(i)}}\right)\left(\prod_{i \leq j<\infty} C_{2}^{\lambda_{j+1-i}^{(i)}}\right) \\
& =\prod_{i=1}^{N}\left(\prod_{k \geq 1} C_{1}^{\lambda_{k}^{(i)}}\right)\left(\prod_{k \geq 1} C_{2}^{\lambda_{k}^{(i)}}\right)=C_{1}^{|\boldsymbol{\lambda}|} C_{2}^{|\boldsymbol{\lambda}|},
\end{aligned}
$$


computing telescoping products in the second step and using $\sum_{i=1}^{N} \sum_{k \geq 1} \lambda_{k}^{(i)}=|\boldsymbol{\lambda}|$ in the last step. This proves the second estimate in (3.13).

To conclude, we prove that the series $\sum_{\boldsymbol{\lambda} \in \mathrm{P}^{N}} \alpha^{|\boldsymbol{\lambda}|}$ for $|\alpha|<1$ is absolutely convergent by the following computation,

$$
\sum_{\lambda \in \mathrm{P}^{N}} \alpha^{|\boldsymbol{\lambda}|}=\sum_{\lambda^{(1)}, \ldots, \lambda^{(N)} \in \mathrm{P}} \prod_{i=1}^{N} \alpha^{\left|\lambda^{(i)}\right|}=\prod_{i=1}^{N} \sum_{\lambda^{(i)} \in \mathrm{P}} \alpha^{\left|\lambda^{(i)}\right|}=\left(\sum_{\lambda \in \mathrm{P}} \alpha^{|\lambda|}\right)^{N}=\frac{1}{(\alpha ; \alpha)_{\infty}^{N}},
$$

using the definition $|\lambda| \equiv \sum_{k \geq 1} \lambda_{k}$ for partitions $\lambda$; for clarity, and for the convenience of the reader, we give in Appendix D the well-known identity used in the last step, together with its elementary proof making absolute convergence manifest; see (D.1)-(D.2).

\section{$4 \mathcal{T}$-operators}

For fixed $N \in \mathbb{Z}_{\geq 1}$, we define an operator $\mathcal{T}$ which acts diagonally on the asymptotically free solution of the trigonometric Ruijsenaars model (Section 4.1). We also present a natural non-stationary generalization of this operator which, as we conjecture, acts diagonally on the corresponding non-stationary Ruijsenaars function (Section 4.2).

\subsection{Trigonometric case}

We find it convenient to work with formal power series.

Definition 4.1. For

$$
\Delta \equiv \sum_{i=1}^{N}\left(x_{i} \partial_{x_{i}}+(N-i) \beta\right)^{2}
$$

with $\beta=\log (t) / \log (q)$, let

$$
\mathcal{T}_{N}(x \mid q, t) \equiv \sum_{\theta \in \mathrm{M}_{N}} \prod_{1 \leq i<j \leq N}\left(x_{j} / x_{i}\right)^{\theta_{i j}} q^{\frac{1}{2} \Delta} c_{N}(\theta|x| q, t) \prod_{1 \leq i<j \leq N} \frac{\left(x_{j} / x_{i} ; q\right)_{\infty}}{\left(t x_{j} / x_{i} ; q\right)_{\infty}}
$$

on $x^{\lambda} \mathbb{C}\left[\left[x_{2} / x_{1}, \ldots, x_{N} / x_{N-1}\right]\right]$ for $\lambda \in \mathbb{C}^{N}$, with $\mathrm{M}_{N}$ in (2.4) and $c_{N}(\theta|s| q, t)$ in (2.5).

Clearly, the operator $\mathcal{T}_{N}(x \mid q, t)$ is complicated: it has the same complexity as the function $f_{N}(x|s| q, t)$; cf. (2.3). Still, it is interesting since, different from the elliptic MacdonaldRuijsenaars operators in (2.8), we know its natural generalization to the non-stationary case; see Section 4.2.

The following is our main result in this section.

Proposition 4.2. The $\mathcal{T}$-operator in (4.2) is well-defined, it commutes with the trigonometric Macdonald-Ruijsenaars operators in (2.1):

$$
\left[\mathcal{T}_{N}(x \mid q, t), D_{N}^{ \pm}(x \mid q, t)\right]=0
$$

on $x^{\lambda} \mathbb{C}\left[\left[x_{2} / x_{1}, \ldots, x_{N} / x_{N-1}\right]\right]$ for all $\lambda \in \mathbb{C}^{N}$, and it acts diagonally on the asymptotically free solutions of the trigonometric Ruijsenaars model in (2.3)-(2.5):

$$
\begin{aligned}
& \mathcal{T}_{N}(x \mid q, t) x^{\lambda} f_{N}(x|s| q, t)=\varepsilon_{N}(s \mid q) x^{\lambda} f_{N}(x|s| q, t), \quad s_{i}=t^{N-i} q^{\lambda_{i}}, \\
& \varepsilon_{N}(s \mid q)=q^{\frac{1}{2} \sum_{i=1}^{N}\left[\log \left(s_{i}\right) / \log (q)\right]^{2}}
\end{aligned}
$$

(note that $\left.\log \left(s_{i}\right) / \log (q)=\lambda_{i}+\beta(N-i)\right)$. 
(A proof based on results in the rest of this section can be found in Appendix C.)

Our proof of Proposition 4.2 is based on the following convenient representation of the $\mathcal{T}$-operator.

Lemma 4.3. For $f(x) \in \mathbb{C}\left[\left[x_{2} / x_{1}, \ldots, x_{N} / x_{N-1}\right]\right]$ and $\lambda \in \mathbb{C}^{N}$,

$$
\mathcal{T}_{N}(x \mid q, t) x^{\lambda} f(x)=\varepsilon(\lambda) x^{\lambda}\left[\prod_{i=1}^{N} \vartheta_{3}\left(s_{i} x_{i} / y_{i} \mid q\right) \chi_{N}(x|y| q, t) \prod_{1 \leq i<j \leq N}\left(1-y_{j} / y_{i}\right) f(y)\right]_{1, y}
$$

with

$$
\begin{gathered}
\varepsilon(\lambda)=q^{\frac{1}{2} \sum_{i}^{N}\left(\lambda_{i}+(N-i) \beta\right)^{2}} \\
\vartheta_{3}(z \mid q) \equiv \sum_{n \in \mathbb{Z}} q^{\frac{1}{2} n^{2}} z^{n} \text { the third Jacobi theta function, } \\
\chi_{N}(x|y| q, t) \equiv f_{N}(x|y| q, t) \prod_{1 \leq i<j \leq N} \frac{\left(q y_{j} / y_{i} ; q\right)_{\infty}}{\left(t y_{j} / y_{i} ; q\right)_{\infty}}
\end{gathered}
$$

and $[\cdots]_{1, y}$ is the constant term in $y$, i.e., for formal Laurent series $g(y)=\sum_{\mu \in \mathbb{Z}^{N}} g_{\mu} y^{\mu}$ as in (4.6), $[g(y)]_{1, y}=g_{0}$.

Remark 4.4. We use $[\cdots]_{1, y}$ only for $g(y) \in \prod_{i=1}^{N} \vartheta_{3}\left(s_{i} x_{i} / y_{i} \mid q\right) \mathbb{C}\left[\left[y_{2} / y_{1}, \ldots, y_{N} / y_{N-1}\right]\right]$, and our definition of $[\cdots]_{1, y}$ is non-ambiguous for these.

Proof of Lemma 4.3. We use that $\mathbb{C}\left[\left[x_{2} / x_{1}, \ldots, x_{N} / x_{N-1}\right]\right]$ is spanned by (a subset of) monomials $x^{\mu}$ with $\mu \in \mathbb{Z}^{N}$. For fixed $\lambda \in \mathbb{C}^{N}$, we compute the action of $q^{\frac{1}{2} \Delta}$ on $x^{\lambda} x^{\mu}, \mu \in \mathbb{Z}^{N}$ :

$$
q^{\frac{1}{2} \Delta} x^{\lambda} x^{\mu}=q^{\frac{1}{2} \sum_{i=1}^{N}\left(\lambda_{i}+\mu_{i}+(N-i) \beta\right)^{2}} x^{\lambda+\mu}=\varepsilon(\lambda) x^{\lambda} \prod_{i=1}^{N}\left(x_{i} s_{i}\right)^{\mu_{i}} q^{\frac{1}{2} \sum_{i=1}^{N} \mu_{i}^{2}}
$$

equal to

$$
\varepsilon(\lambda) x^{\lambda}\left[\prod_{i=1}^{N} \vartheta_{3}\left(s_{i} x_{i} / y_{i} \mid q\right) y^{\mu}\right]_{1, y},
$$

and thus

$$
q^{\frac{1}{2} \Delta} x^{\lambda} f(x)=\varepsilon(\lambda) x^{\lambda}\left[\prod_{i=1}^{N} \vartheta_{3}\left(s_{i} x_{i} / y_{i} \mid q\right) f(y)\right]_{1, y}
$$

for all $f(x) \in \mathbb{C}\left[\left[x_{2} / x_{1}, \ldots, x_{N} / x_{N-1}\right]\right]$. This and the definition in (4.2) give

$$
\begin{aligned}
\mathcal{T}_{N}(x \mid q, t) x^{\lambda} f(x)= & \varepsilon(\lambda) x^{\lambda}\left[\prod_{i=1}^{N} \vartheta_{3}\left(s_{i} x_{i} / y_{i} \mid q\right)\right. \\
& \left.\times \sum_{\theta \in \mathrm{M}_{N}} \prod_{1 \leq i<j \leq N}\left(x_{j} / x_{i}\right)^{\theta_{i j}} c_{N}(\theta|y| q, t) \prod_{1 \leq i<j \leq N} \frac{\left(y_{j} / y_{i} ; q\right)_{\infty}}{\left(t y_{j} / y_{i} ; q\right)_{\infty}} f(y)\right]_{1, y},
\end{aligned}
$$

and using (2.3) and the definition in (4.7) we obtained (4.6).

We note that $\chi_{N}(x|y| q, t)=\chi_{N}(y|x| q, q / t)$ (this is proved in Appendix C, Lemma C.1); inserting this in (4.6) and backtracking, one obtains the following alternative representation of the $\mathcal{T}$-operator:

$$
\mathcal{T}_{N}(x \mid q, t) \equiv \frac{\left(q x_{j} / x_{i} ; q\right)_{\infty}}{\left(q x_{j} / t x_{i} ; q\right)_{\infty}} \sum_{\theta \in \mathrm{M}_{N}} c_{N}(\theta|x| q, q / t) q^{\frac{1}{2} \Delta} \prod_{1 \leq i<j \leq N}\left(x_{j} / x_{i}\right)^{\theta_{i j}} \prod_{1 \leq i<j \leq N}\left(1-x_{j} / x_{i}\right) .
$$




\subsection{Non-stationary case}

We present a non-stationary generalization of the $\mathcal{T}$-operator.

Definition 4.5. For $\Delta$ in (4.1) with $\beta=\log (t) / \log (q)$, let

$$
\mathcal{T}_{N, \infty}(x, p \mid q, t, \kappa)=\sum_{\theta \in \hat{\mathrm{M}}_{N}} \prod_{i=1}^{N} \prod_{j>i}\left(x_{j} / x_{i}\right)^{\theta_{i j}} q^{\frac{1}{2} \Delta} T_{\kappa, p} c_{N, \infty}(\theta|\bar{x}| q, t) \prod_{i=1}^{N} \prod_{j>i} \frac{\left(x_{j} / x_{i} ; q\right)_{\infty}}{\left(t x_{j} / x_{i} ; q\right)_{\infty}}
$$

with $x_{i+N}=p x_{i}$ for $i \geq 1$ on $x^{\lambda} \mathbb{C}\left[\left[x_{2} / x_{1}, x_{3} / x_{2}, \ldots, x_{N} / x_{N-1}, p x_{1} / x_{N}\right]\right]$ for $\lambda \in \mathbb{C}^{N}$, with $\hat{\mathrm{M}}_{N}$ in $(3.2)$ and $c_{N, \infty}(\theta|\bar{x}| q, t)$ in (3.3).

Remark 4.6. To make the $p$-dependence of this operator manifest, one can write it as

$$
\begin{aligned}
\mathcal{T}_{N, \infty}(x, p \mid q, t, \kappa)= & \sum_{\theta \in \hat{\mathrm{M}}_{N}} e_{N, \infty}(x, p) q^{\frac{1}{2} \Delta} T_{\kappa, p} c_{N, \infty}(\theta|x, p| q, t) \\
& \times \prod_{1 \leq i<j \leq N} \frac{\left(x_{j} / x_{i} ; q, p\right)_{\infty}}{\left(t x_{j} / x_{i} ; q, p\right)_{\infty}} \prod_{1 \leq i \leq j \leq N} \frac{\left(p x_{i} / x_{j} ; q, p\right)_{\infty}}{\left(p t x_{i} / x_{j} ; q, p\right)_{\infty}}
\end{aligned}
$$

using the definitions in (3.6) and Lemma D.1 in Appendix D.

By comparing with (3.1)-(3.3), it is clear that the operator in (4.9) is a natural non-stationary generalization of the trigonometric $\mathcal{T}$-operators in (4.2); however, there is one important new feature: the shift operator $T_{\kappa, p}$ acting on $p$.

We propose the following generalization to Proposition 4.2 ; this conjecture is a complement to the ones in [21].

Conjecture 4.7. The non-stationary $\mathcal{T}$-operator in (4.9) has a well-defined diagonal action on the non-stationary Ruijsenaars function in Definitions 2.1 and 3.3:

$$
\mathcal{T}_{N, \infty}(x, p \mid q, t, \kappa) x^{\lambda} f_{N, \infty}(x, p|s, \kappa| q, t)=\varepsilon_{N}(s \mid q) x^{\lambda} f_{N, \infty}(x, p|s, \kappa| q, t), \quad s_{i}=t^{N-i} q^{\lambda_{i}}
$$

with $\varepsilon_{N}(s \mid q)$ given in (4.5).

In the rest of this section, we present two generalizations of results about the trigonometric $\mathcal{T}$-operators: (i) the constant-term representation of the $\mathcal{T}$-operator in Lemma 4.3(ii) the alternative representation in (4.8) obtained with the duality in (2.12). We also rephrase Conjecture 4.7 in terms of the non-stationary Ruijsenaars functions as defined in [21].

One can adapt the proof Lemma 4.3 to obtain the following constant-term representation of the $\mathcal{T}$-operator in (4.9):

Lemma 4.8. For $f(x, p) \in \mathbb{C}\left[\left[x_{2} / x_{1}, p x_{3} / x_{2}, \ldots, x_{N} / x_{N-1}, p x_{1} / x_{N}\right]\right]$ and $\lambda \in \mathbb{C}^{N}$,

$$
\begin{aligned}
& \mathcal{T}_{N, \infty}(x, p \mid q, t, \kappa) x^{\lambda} f(x, p) \\
& \quad=\varepsilon(\lambda) x^{\lambda}\left[\prod_{i=1}^{N} \vartheta_{3}\left(s_{i} x_{i} / y_{i} \mid q\right) \frac{1}{1-\kappa p / u} \chi_{N, \infty}(x, p|y, u| q, t) \prod_{i=1}^{N} \prod_{j>i}\left(1-y_{j} / y_{i}\right) f(y, u)\right]_{1, y, u}
\end{aligned}
$$

with

$$
\chi_{N, \infty}(x, p|y, u| q, t)=f_{N, \infty}(x, p|y, u| q, t) \prod_{i=1}^{N} \prod_{j>i} \frac{\left(q y_{j} / y_{i} ; q\right)_{\infty}}{\left(t y_{j} / y_{i} ; q\right)_{\infty}}
$$

setting $y_{i+N}=u y_{i}$ for all $i \geq 1, f_{N, \infty}(x, p|y, u| q, t)$ in $(3.1)-(3.3)$, and $[\cdots]_{1 ; y, u}$, short for $\left[[\cdots]_{1, y}\right]_{1, u}$, the constant term in $y \in \mathbb{C}^{N}$ and $u \in \mathbb{C}$. 
Proof. This is proved by a straightforward generalization of the arguments given in the proof of Lemma 4.3; the only new ingredient is

$$
T_{\kappa, p} p^{n}=(\kappa p)^{n}=\left[\frac{1}{1-\kappa p / u} u^{n}\right]_{1, u} \quad\left(n \in \mathbb{Z}_{\geq 0}\right),
$$

and therefore

$$
q^{\frac{1}{2} \Delta} T_{\kappa, p} x^{\lambda} f(x, p)=\varepsilon(\lambda) x^{\lambda}\left[\prod_{i=1}^{N} \vartheta_{3}\left(s_{i} x_{i} / y_{i} \mid q\right) \frac{1}{1-\kappa p / u} f(y, u)\right]_{1, y, u}
$$

for all $f(x, p) \in \mathbb{C}\left[\left[x_{2} / x_{1}, p x_{3} / x_{2}, \ldots, x_{N} / x_{N-1}, p x_{1} / x_{N}\right]\right]$.

Moreover, by an argument similar to the one for the trigonometric $\mathcal{T}$-operator above, the conjectured duality in (3.10) implies

$$
\chi_{N, \infty}(x, p|y, u| q, t)=\chi_{N, \infty}(y, u|x, p| q, q / t)
$$

and the following alternative representation of this $\mathcal{T}$-operator

$$
\begin{aligned}
\mathcal{T}_{N, \infty}(x, p \mid q, t, \kappa)= & \prod_{i=1}^{N} \prod_{j>i} \frac{\left(q x_{j} / x_{i} ; q\right)_{\infty}}{\left(q x_{j} / t x_{i} ; q\right)_{\infty}} \sum_{\theta \in \hat{\mathcal{M}}_{N}} c_{N, \infty}(\theta|\bar{x}| q, t) q^{\frac{1}{2} \Delta} T_{\kappa, p} \\
& \times \prod_{i=1}^{N} \prod_{j>i}\left(x_{j} / x_{i}\right)^{\theta_{i j}} \prod_{i=1}^{N} \prod_{j>i}\left(1-x_{j} / x_{i}\right)
\end{aligned}
$$

with $x_{i+N}=p x_{i}$ for all $i \geq 1$.

To conclude, we rephrase Conjecture 4.7 using balanced coordinates.

Definition 4.9. For $\Delta$ as in (4.1) with $\beta=\log (q / t) / \log (q)$, let

$$
\begin{aligned}
\mathcal{T}^{\widehat{\mathfrak{g}} \mathrm{I}_{N}}(x, p \mid q, t, \kappa) & \sum_{\lambda^{(1)}, \ldots, \lambda^{(N)} \in \mathrm{P}} \prod_{\beta=1}^{N} \prod_{\alpha \geq 1}\left(p x_{\alpha+\beta} / t x_{\alpha+\beta-1}\right)^{\lambda_{\alpha}^{(\beta)}} q^{\frac{1}{2} \Delta^{\Delta}} T_{\kappa, p} \prod_{i, j=1}^{N} \frac{\mathrm{N}_{\lambda^{(i)}, \lambda^{(j)}}^{(j-i \mid N)}\left(t x_{j} / x_{i} \mid q, p\right)}{\mathrm{N}_{\lambda^{(i)}, \lambda^{(j)}}^{(j-i)}\left(x_{j} / x_{i} \mid q, p\right)} \\
& \times \prod_{1 \leq i<j \leq N} \frac{\left(p^{j-i} x_{j} / x_{i} ; q, p^{N}\right)_{\infty}}{\left(p^{j-i} q x_{j} / t x_{i} ; q, p^{N}\right)_{\infty}} \prod_{1 \leq i \leq j \leq N} \frac{\left(p^{N-j+i} x_{i} / x_{j} ; q, p^{N}\right)_{\infty}}{\left(p^{N-j+i} q x_{i} / t x_{j} ; q, p^{N}\right)_{\infty}}
\end{aligned}
$$

with $x_{i+N}=x_{i}$ for $i \geq 1$ on $x^{\lambda} \mathbb{C}\left[\left[p x_{2} / x_{1}, p x_{3} / x_{2}, \ldots, p x_{N} / x_{N-1}, p x_{1} / x_{N}\right]\right]$ for $\lambda \in \mathbb{C}^{N}$, with $\mathrm{P}$ the set of all partitions and $\mathrm{N}_{\lambda, \mu}^{(k \mid N)}(u \mid q, \kappa)$ the Nekrasov factors given in (2.10).

Fact 4.10. Conjecture 4.7 is equivalent to the following diagonal action of the non-stationary $\mathcal{T}$-operator in (4.10) on the non-stationary Ruijsenaars function in $(2.9)-(2.10)$,

$$
\mathcal{T}^{\widehat{\mathfrak{g}} \mathfrak{l}_{N}}(x, p \mid q, t) x^{\lambda} f^{\widehat{\mathfrak{g} l} l_{N}}(x, p|s, \kappa| q, t)=\varepsilon_{N}(s \mid q) x^{\lambda} f^{\widehat{\mathfrak{g l}} l_{N}}(x, p|s, \kappa| q, t), \quad s_{i}=(q / t)^{N-i} q^{\lambda_{i}} .
$$

Proof. This is implied by Theorem 3.2, using that the shift operator $T_{\kappa, p}$ commutes with the following operator, $\Phi$, switching from unbalanced to balanced coordinates:

$$
(\Phi f)(x, p|s, \kappa| q, t) \equiv f\left(p^{-\delta} x, p^{N}\left|\kappa^{-\delta} s, \kappa^{N}\right| q, t / q\right),
$$

and noting that Theorem 3.2 implies $\left(\Phi f_{N, \infty}\right)(x, p|s, \kappa| q, t)=f^{\widehat{\mathfrak{g} l} l_{N}}(x, p|s, \kappa| q, t)$. 


\section{Final remarks}

The main conjecture in [21, Conjecture 1.14] can be tested systematically using a perturbative solution of the elliptic Ruijsenaars model that generalizes the perturbative solution of the elliptic Calogero-Sutherland (eCS) model in [12]. We plan to present this elsewhere.

As already mentioned, one important outstanding problem is to find $\kappa$-deformations of the elliptic Ruijsenaars operators in (2.8) that have the non-stationary Ruijsenaars functions as eigenfunctions. As conjectured in [21], the limit $q \rightarrow 1$ of this hypothetical non-stationary Ruijsenaars model is a known non-stationary eCS model depending on parameters $\beta, p$ and $\kappa$ related to the non-stationary Ruijsenaars parameters as follows, $t=q^{\beta}$ and $\kappa=q^{-\varkappa}{ }^{6}$ Recently, a rigorous construction of integral representations of eigenfunctions of the non-stationary eCS model for $\varkappa=\beta$ was presented [2]. We hope that, by combining the latter results with recent results on the non-stationary Ruijsenaars functions for the corresponding special value of $\kappa$ [9], it will be possible to prove the main conjecture in [21] in the non-stationary eCS limit $q \rightarrow 1$ and for $\varkappa=\beta$. Another possible strategy to prove the conjecture in [21] for $q \rightarrow 1$ and general $\varkappa$-values is to try to generalize the perturbative solution of the non-stationary Lamé equation in [1] (note that the latter equation reduces to the non-stationary eCS model for $N=2$ in special cases).

The elliptic Ruijsenaars model is invariant under the exchange $p \leftrightarrow q$ [17]. ${ }^{7}$ The non-stationary Ruijsenaars functions do not have this property manifest; we plan to report elsewhere on how this duality is recovered from the non-stationary Ruijsenaars function.

It was suggested more than 20 years ago that the elliptic Ruijsenaars model has a doubleelliptic generalization with remarkable duality properties [4, 8], and recently an explicit formula for an operator defining such a model was conjectured [11]. It would be interesting to obtain a better understanding of the relation between the non-stationary Ruijsenaars functions and this double elliptic system recently proposed in [3].

Since the non-stationary $\mathcal{T}$-operator proposed in this paper contains a factor $q^{\frac{1}{2} \Delta} T_{\kappa, p}$, its eigenvalue equation can be regarded as a $q$-deformed heat equation. We mention the work of Felder and Varchenko on the $q$-deformed KZB heat equation [5, 6] which seems related; it would be interesting to understand this relation in detail.

\section{A Alternative series representation}

We prove Theorem 3.2. We start with details complementing the concise proof of Lemma 3.5 in the main text (Appendix A.1). The main part of the proof is in Appendix A.2.

\section{A.1 Details on Lemma 3.5}

One can check that the following two formulas provide a correspondence between multi-partitions $\boldsymbol{\lambda}=\left(\lambda^{(1)}, \ldots, \lambda^{(N)}\right)$ in $\mathrm{P}^{N}$ and matrices $\theta=\left(\theta_{i k}\right)_{i, k=1}^{\infty}$ in $\hat{\mathrm{M}}_{N}$ that is one-to-one:

$$
\theta_{i k}=\lambda_{k-i}^{(i)}-\lambda_{k-i+1}^{(i)}
$$

and

$$
\lambda_{k-i}^{(i)}=\sum_{a \geq k} \theta_{i a}
$$

\footnotetext{
${ }^{6}$ The elliptic deformation parameter $p$ is the same in both cases.

${ }^{7}$ We thank S. Ruijsenaars for pointing this out at the NORDITA workshop "Elliptic integrable systems, special functions and quantum field theory" in June 2019.
} 
setting $\lambda^{(i+N)}=\lambda^{(i)}$ for all $i \geq 1$. With this identification, $C_{N, \infty}(\boldsymbol{\lambda}|\bar{s}| q, t)$ in (3.9) is clearly equal to $c_{N, \infty}(\theta|\bar{s}| q, t)$ in $(3.3)$, and

$$
\begin{aligned}
\prod_{i=1}^{N} \prod_{k=i+1}^{\infty}\left(x_{k} / x_{i}\right)^{\theta_{i k}} & =\prod_{i=1}^{N} \prod_{k=i+1}^{\infty}\left[\left(x_{k} / x_{k-1}\right)\left(x_{k-1} / x_{k-2}\right) \cdots\left(x_{i+1} / x_{i}\right)\right]^{\theta_{i k}} \\
& =\prod_{i=1}^{N} \prod_{i<j \leq k<\infty}\left(x_{j} / x_{j-1}\right)^{\lambda_{k-i}^{(i)}-\lambda_{k-i+1}^{(i)}}=\prod_{i=1}^{N} \prod_{j>i}\left(x_{j} / x_{j-1}\right)^{\lambda_{j-i}^{(i)}} \\
& =\prod_{i=1}^{N} \prod_{k \geq 1}\left(x_{i+k} / x_{i+k-1}\right)^{\lambda_{k}^{(i)}},
\end{aligned}
$$

inserting a telescoping product in the second step, using (A.1) in the third, and computing a telescoping product in the fourth. This proves the result.

\section{A.2 Proof of Theorem 3.2}

We show by direct computations that the function on the left-hand side in (3.7) is equal to the function $f_{N, \infty}(\bar{x}|\bar{s}| q, t)$ in (3.8)-(3.9) with $x_{i+N}=p x_{i}$ and $s_{i+N}=\kappa s_{i}$, for all $i \geq 1$. This, together with Lemma 3.5, proves the result.

We compute the function on the left-hand side in (3.7) using (2.9)-(2.10):

$$
\begin{aligned}
f^{\widehat{\mathfrak{g}}{ }_{N}} & \left(p^{\delta / N} x, p^{1 / N}\left|\kappa^{\delta / N} s, \kappa^{1 / N}\right| q, q / t\right) \\
= & \sum_{\lambda^{(1)}, \ldots, \lambda^{(N)} \in \mathrm{P}} \prod_{i, j=1}^{N} \frac{\mathrm{N}_{\lambda^{(i)}, \lambda^{(j)}}^{(j-i \mid N)}\left((q / t)\left(\kappa^{\delta / N} s\right)_{j} /\left(\kappa^{\delta / N} s\right)_{i} \mid q, \kappa^{1 / N}\right)}{\mathrm{N}_{\lambda^{(i)}, \lambda^{(j)}}^{(j-i \mid N)}\left(\left(\kappa^{\delta / N} s\right)_{j} /\left(\kappa^{\delta / N} s\right)_{i} \mid q, \kappa\right)} \\
& \times \prod_{\beta=1}^{N} \prod_{\alpha \geq 1}\left(p^{1 / N}\left(p^{\delta / N}\right)_{\alpha+\beta} t / q\left(p^{\delta / N}\right)_{\alpha+\beta-1}\right)^{\lambda_{\alpha}^{(\beta)}} .
\end{aligned}
$$

By Definition 2.1 of the non-stationary Ruijsenaars functions, the variables $\left(p^{\delta / N} x\right)_{i}$ above are extended from $i=1, \ldots, N$ to all $i \geq 1$ by the rule $\left(p^{\delta / N} x\right)_{i+N}=\left(p^{\delta / N} x\right)_{i}$, whereas $x_{i+N}=p x_{i}$ for all $i \geq 1$ implies $\left(p^{\delta / N} x\right)_{i+k N}=p^{(N-i) / N} x_{i}=p^{[N-(i+k N)] / N} x_{i+k N}$ for all $i=1, \ldots, N$ and $k \in \mathbb{Z}_{\geq 1}$, and thus

$$
\left(p^{\delta / N} x\right)_{i}=p^{(N-i) / N} x_{i} \quad(i \geq 1) .
$$

Therefore,

$$
\begin{aligned}
& \prod_{\beta=1}^{N} \prod_{\alpha \geq 1}\left(p^{1 / N}\left(p^{\delta / N} x\right)_{\alpha+\beta} t / q\left(p^{\delta / N} x\right)_{\alpha+\beta-1}\right)^{\lambda_{\alpha}^{(\beta)}} \\
& \quad=\prod_{\beta=1}^{N} \prod_{\alpha \geq 1}\left(p^{1 / N} p^{(N-\alpha-\beta) / N} x_{\alpha+\beta} t / q p^{(N-\alpha-\beta+1) / N} x_{\alpha+\beta-1}\right)^{\lambda_{\alpha}^{(\beta)}} \\
& =(t / q)^{|\lambda|} \prod_{\beta=1}^{N} \prod_{\alpha \geq 1}\left(x_{\alpha+\beta} / x_{\alpha+\beta-1}\right)^{\lambda_{\alpha}^{(\beta)}}
\end{aligned}
$$

using the abbreviation $|\boldsymbol{\lambda}| \equiv \sum_{\beta=1}^{N} \sum_{\alpha \geq 1} \lambda_{\alpha}^{(\beta)}$. Renaming indices $(\alpha, \beta) \rightarrow(k, i)$, we thus can write the function on the left-hand side in (3.7) as

$$
f^{\widehat{\mathfrak{g}}_{N}}\left(p^{\delta / N} x, p^{1 / N}\left|\kappa^{\delta / N} s, \kappa^{1 / N}\right| q, q / t\right)=\sum_{\boldsymbol{\lambda} \in \mathrm{P}^{N}} \tilde{C}_{N}(\boldsymbol{\lambda} ; s \mid q, t, \kappa) \prod_{i=1}^{N} \prod_{k \geq 1}\left(x_{i+k} / x_{i+k-1}\right)^{\lambda_{k}^{(i)}}
$$


with $x_{i+N}=p x_{i}$ for all $i \geq 1$ and

$$
\tilde{C}_{N}(\boldsymbol{\lambda} ; s \mid q, t, \kappa)=(t / q)^{|\boldsymbol{\lambda}|} \prod_{i, j=1}^{N} \frac{\mathrm{N}_{\lambda^{(i)}, \lambda^{(j)}}^{(j-i \mid N)}\left((q / t)\left(\kappa^{\delta / N} s\right)_{j} /\left(\kappa^{\delta / N} s\right)_{i} \mid q, \kappa^{1 / N}\right)}{\mathrm{N}_{\lambda^{(i)}, \lambda^{(j)}}^{(j-i \mid N)}\left(\left(\kappa^{\delta / N} s\right)_{j} /\left(\kappa^{\delta / N} s\right)_{i} \mid q, \kappa^{1 / N}\right)} .
$$

To complete the proof, we have to show that $\tilde{C}_{N}(\boldsymbol{\lambda} ; s \mid q, t, \kappa)$ in (A.2) is equal to $C_{N, \infty}(\boldsymbol{\lambda} ; \bar{s} \mid q, t)$ in (3.9) for $s_{i+N}=\kappa s_{i}(i \geq 1)$. For that, we compute the Nekrasov factors in (2.10), partially specializing to the variables we need:

$$
\begin{aligned}
\mathrm{N}_{\lambda^{(i)}, \lambda(j)}^{(j-i \mid N)}\left(u \mid q, \kappa^{1 / N}\right)= & \prod_{\substack{b \geq a \geq 1 \\
b-a \equiv(j-i)(\bmod N)}}\left(u q^{-\lambda_{a}^{(j)}+\lambda_{b+1}^{(i)}} \kappa^{(-a+b) / N} ; q\right)_{\lambda_{b}^{(i)}-\lambda_{b+1}^{(i)}} \\
& \times \prod_{\substack{\beta \geq \alpha \geq 1 \\
\beta-\alpha \equiv(i-j-1)(\bmod N)}}\left(u q^{\lambda_{\alpha}^{(i)}-\lambda_{\beta}^{(j)}} \kappa^{(\alpha-\beta-1) / N} ; q\right)_{\lambda_{\beta}^{(j)}-\lambda_{\beta+1}^{(j)}} .
\end{aligned}
$$

We note that the constraints on $b$ in the first product is solved by $b=a+j+\ell N-i$ with $\ell$ an arbitrary integer $\geq \chi(i>j)$, using the definition $\chi(i>j)=1$ for $j<i$ and 0 otherwise; similarly, the constraints on $\beta$ in the second product is solved by $\beta=\alpha+i+\ell^{\prime} N-j-1$ with arbitrary integer $\ell^{\prime} \geq \chi(j \geq i)$. We thus can write these Nekrasov factors as

$$
\begin{aligned}
& \mathrm{N}_{\lambda^{(i)}, \lambda(j)}^{(j-i \mid N)}\left(u \mid q, \kappa^{1 / N}\right)=\prod_{a \geq 1} \prod_{\ell \geq \chi(i>j)}\left(u q^{-\lambda_{a}^{(j)}+\lambda_{a+j+\ell N-i+1}^{(i)}} \kappa^{(j+\ell N-i) / N} ; q\right)_{\lambda_{a+j+\ell N-i}^{(i)}-\lambda_{a+j+\ell N-i+1}^{(i)}} \\
& \times \prod_{\alpha \geq 1} \prod_{\ell^{\prime} \geq \chi(j \geq i)}\left(u q^{\lambda_{\alpha}^{(i)}-\lambda_{\alpha+i+\ell^{\prime} N-j-1}^{(j)}} \kappa^{\left(j-i-\ell^{\prime} N\right) / N} ; q\right)_{\lambda_{\alpha+i+\ell^{\prime} N-j-1}^{(j)}-\lambda_{\alpha+i+\ell^{\prime} N-j}^{(j)}} .
\end{aligned}
$$

We now specialize further to the arguments of interest to us:

$$
u=c\left(\kappa^{\delta / N} s\right)_{j} /\left(\kappa^{\delta / N} s\right)_{i}=c \kappa^{(N-j) / N} s_{j} / \kappa^{(N-i) / N} s_{i}=c \kappa^{(i-j) / N} s_{j} / s_{i}, \quad c \in\{1, q / t\} .
$$

For these arguments, the manifest $\kappa$-dependence disappears:

$$
\begin{aligned}
\mathrm{N}_{\lambda^{(i)}, \lambda^{(j)}}^{(j-i \mid N)}\left(c\left(\kappa^{\delta / N} s\right)_{j} /\left(\kappa^{\delta / N} s\right)_{i} \mid q, \kappa^{1 / N}\right) \\
=\prod_{a \geq 1} \prod_{\ell \geq \chi(i>j)}\left(c q^{-\lambda_{a}^{(j)}+\lambda_{a+j+\ell N-i+1}^{(i)}} s_{j+\ell N} / s_{i} ; q\right)_{\lambda_{a+j+\ell N-i}^{(i)}-\lambda_{a+j+\ell N-i+1}^{(i)}} \\
\quad \times \prod_{\alpha \geq 1} \prod_{\ell^{\prime} \geq \chi(j \geq i)}\left(c q^{\lambda_{\alpha}^{(i)}-\lambda_{\alpha+i+\ell^{\prime} N-j-1}^{(j)}} s_{j} / s_{i+\ell^{\prime} N} ; q\right)_{\lambda_{\alpha+i+\ell^{\prime} N-j-1}^{(j)}-\lambda_{\alpha+i+\ell^{\prime} N-j}^{(j)}}
\end{aligned}
$$

using $\kappa^{\ell} s_{j} / s_{i}=s_{j+\ell N} / s_{i}$ and $\kappa^{-\ell^{\prime}} s_{j} / s_{i}=s_{j} / s_{i+\ell^{\prime} N}$ implied by $s_{i+N}=\kappa s_{i}$ for $i \geq 1$. We now take the product of these Nekrasov factors over $i, j=1, \ldots, N$, change variables $j+\ell N \rightarrow j$ in the first group of products and $i+\ell^{\prime} N \rightarrow i$ in the second group, and obtain

$$
\begin{aligned}
\prod_{i, j=1}^{N} \mathrm{~N}_{\lambda^{(i)}, \lambda^{(j)}}^{(j-i \mid N)}\left(c\left(\kappa^{\delta / N} s\right)_{j} /\left(\kappa^{\delta / N} s\right)_{i} \mid q, \kappa^{1 / N}\right) \\
=\prod_{i=1}^{N} \prod_{j \geq i}\left(c q^{-\lambda_{a}^{(j)}+\lambda_{a+j-i+1}^{(i)}} s_{j} / s_{i} ; q\right)_{\lambda_{a+j-i}^{(i)}-\lambda_{a+j-i+1}^{(i)}} \\
\quad \times \prod_{j=1}^{N} \prod_{i>j} \prod_{\alpha \geq 1}\left(c q^{\lambda_{\alpha}^{(i)}-\lambda_{\alpha+i-j-1}^{(j)}} s_{j} / s_{i} ; q\right)_{\lambda_{\alpha+i-j-1}^{(j)}-\lambda_{\alpha+i-j}^{(j)}}
\end{aligned}
$$




$$
\begin{aligned}
= & \prod_{i=1}^{N} \prod_{j \geq i} \prod_{k>j}\left(c q^{-\lambda_{k-j}^{(j)}+\lambda_{k-i+1}^{(i)}} s_{j} / s_{i} ; q\right)_{\lambda_{k-i}^{(i)}-\lambda_{k-i+1}^{(i)}} \\
& \times \prod_{j=1}^{N} \prod_{i>j} \prod_{k \geq i}\left(c q^{\lambda_{k-i+1}^{(i)}-\lambda_{k-j}^{(j)}} s_{j} / s_{i} ; q\right)_{\lambda_{k-j}^{(j)}-\lambda_{k-j+1}^{(j)}},
\end{aligned}
$$

where we changed variables $a \rightarrow k=a+j$ and $\alpha \rightarrow k=\alpha+i-1$ in the last step. We find it convenient to write this result as

$$
\begin{aligned}
\prod_{i, j=1}^{N} \mathrm{~N}_{\lambda^{(i)}, \lambda^{(j)}}^{(j-i \mid N)}\left(c\left(\kappa^{\delta / N} s\right)_{j} /\left(\kappa^{\delta / N} s\right)_{i} \mid q, \kappa^{1 / N}\right)= & \prod_{i=1}^{N} \prod_{i \leq j<k<\infty}\left(c q^{-\lambda_{k-j}^{(j)}+\lambda_{k-i+1}^{(i)}} s_{j} / s_{i} ; q\right)_{\lambda_{k-i}^{(i)}-\lambda_{k-i+1}^{(i)}} \\
& \times \prod_{i=1}^{N} \prod_{i<j \leq k<\infty}\left(c q^{\lambda_{k-j+1}^{(j)}-\lambda_{k-i}^{(i)}} s_{i} / s_{j} ; q\right)_{\lambda_{k-i}^{(i)}-\lambda_{k-i+1}^{(i)}}
\end{aligned}
$$

swapping variable names $i \leftrightarrow j$ in the second group of products. We insert this into (A.2) to obtain

$$
\begin{aligned}
\tilde{C}_{N}(\boldsymbol{\lambda} ; s \mid q, t, \kappa)= & (t / q)^{|\boldsymbol{\lambda}|} \prod_{i=1}^{N} \prod_{i \leq j<k<\infty} \frac{\left(q^{-\lambda_{k-j}^{(j)}+\lambda_{k-i+1}^{(i)} q s_{j}} / t s_{i} ; q\right)_{\lambda_{k-i}^{(i)}-\lambda_{k-i+1}^{(i)}}}{\left(q^{-\lambda_{k-j}^{(j)}+\lambda_{k-i+1}^{(i)}} s_{j} / s_{i} ; q\right)_{\lambda_{k-i}^{(i)}-\lambda_{k-i+1}^{(i)}}} \\
& \times \prod_{i=1}^{N} \prod_{i<j \leq k<\infty} \frac{\left(q^{\lambda_{k-j+1}^{(j)}-\lambda_{k-i}^{(i)}} q s_{i} / t s_{j} ; q\right)_{\lambda_{k-i}^{(i)}-\lambda_{k-i+1}^{(i)}}}{\left(q^{\lambda_{k-j+1}^{(j)}-\lambda_{k-i}^{(i)}} s_{i} / s_{j} ; q\right)_{\lambda_{k-i}^{(i)}-\lambda_{k-i+1}^{(i)}}}
\end{aligned}
$$

To proceed, we use the well-known identity

$$
\frac{\left(q^{-m} / a ; q\right)_{m}}{\left(q^{-m} / b ; q\right)_{m}}=(b / a)^{m} \frac{(q a ; q)_{m}}{(q b ; q)_{m}} \quad\left(a, b \in \mathbb{C}, m \in \mathbb{Z}_{\geq 0}\right) .
$$

Applying this to the factors in the second group of products for $m=\lambda_{k-i}^{(i)}-\lambda_{k-i+1}^{(i)}, b=$ $q^{\lambda_{k-i+1}^{(i)}-\lambda_{k-j+1}^{(j)}} s_{j} / s_{i}, a=t b / q$ yields

$$
\begin{aligned}
\tilde{C}_{N}(\boldsymbol{\lambda} ; s \mid q, t, \kappa)= & (t / q)^{|\boldsymbol{\lambda}|} \prod_{i=1}^{N} \prod_{i \leq j<k<\infty} \frac{\left(q^{-\lambda_{k-j}^{(j)}+\lambda_{k-i+1}^{(i)}} q s_{j} / t s_{i} ; q\right)_{\lambda_{k-i}^{(i)}-\lambda_{k-i+1}^{(i)}}}{\left(q^{-\lambda_{k-j}^{(j)}+\lambda_{k-i+1}^{(i)}} s_{j} / s_{i} ; q\right)_{\lambda_{k-i}^{(i)}-\lambda_{k-i+1}^{(i)}}} \\
& \times \prod_{i=1}^{N} \prod_{i<j \leq k<\infty}(q / t)^{\lambda_{k-i}^{(i)}-\lambda_{k-i+1}^{(i)}} \frac{\left(q^{\lambda_{k-i+1}^{(i)}-\lambda_{k-j+1}^{(j)}} t s_{j} / s_{i}\right)_{\lambda_{k-i}^{(i)}-\lambda_{k-i+1}^{(i)}}}{\left(q^{\lambda_{k-i+1}^{(i)}-\lambda_{k-j+1}^{(j)}} q s_{j} / s_{i}\right)_{\lambda_{k-i}^{(i)}-\lambda_{k-i+1}^{(i)}}}
\end{aligned}
$$

To complete the proof that $\tilde{C}_{N}(\boldsymbol{\lambda} ; s \mid q, t, \kappa)$ in (A.2) is identical with $C_{N, \infty}(\boldsymbol{\lambda}|\bar{s}| q, t)$ in (3.9), we swap the order of the two groups of products and compute the overall power of $(q / t)$ :

$$
\begin{aligned}
\prod_{i=1}^{N} \prod_{i<j \leq k<\infty}(q / t)^{\lambda_{k-i}^{(i)}-\lambda_{k-i+1}^{(i)}} & =\prod_{i=1}^{N} \prod_{j=i+1}^{\infty} \prod_{k=j}^{\infty}(q / t)^{\lambda_{k-i}^{(i)}-\lambda_{k-i+1}^{(i)}} \\
& =\prod_{i=1}^{N} \prod_{j=i+1}^{\infty}(q / t)^{\lambda_{j-i}^{(i)}}=\prod_{i=1}^{N} \prod_{k \geq 1}(q / t)^{\lambda_{k}^{(i)}}=(q / t)^{|\boldsymbol{\lambda}|},
\end{aligned}
$$

cancelling the factor $(t / q)^{|\boldsymbol{\lambda}|}$. This proves the identity in (3.4) with $f_{N, \infty}(\bar{x}|\bar{s}| q, t)$ in (3.8)-(3.9) and $x_{i+N}=p x_{i}, s_{i+N}=\kappa s_{i}(i \geq 1)$. This, together with Lemma 3.5, implies the result. 


\section{B Estimates}

We give a complementary proof of the second estimate in (3.13), to compute the upper bounds $C_{1,2}$ in Theorem 3.7.

\section{B.1 Complementary proof of the second estimate in (3.13)}

We prove that, under the assumptions in Theorem 3.7, the following estimates hold true for the fractions appearing in the formula (3.9) for $C_{N, \infty}(\boldsymbol{\lambda}|\bar{s}| q, t)$,

$$
\begin{aligned}
& \left|\frac{\left(q^{\lambda_{k-i+1}^{(i)}-\lambda_{k-j+1}^{(j)}} t s_{j} / s_{i} ; q\right)_{\lambda_{k-i}^{(i)}-\lambda_{k-i+1}^{(i)}}}{\left(q^{\lambda_{k-i+1}^{(i)}-\lambda_{k-j+1}^{(j)} q s_{j}} / s_{i} ; q\right)_{\lambda_{k-i}^{(i)}-\lambda_{k-i+1}^{(i)}}}\right| \leq C_{1}^{\lambda_{k-i}^{(i)}-\lambda_{k-i+1}^{(i)}} \quad(1 \leq i \leq N, i<j \leq k<\infty), \\
& \left|\frac{\left(q^{-\lambda_{k-j}^{(j)}+\lambda_{k-i+1}^{(i)} q s_{j}} / t s_{i} ; q\right)_{\lambda_{k-i}^{(i)}-\lambda_{k-i+1}^{(i)}}}{\left(q^{-\lambda_{k-j}^{(j)}+\lambda_{k-i+1}^{(i)}} s_{j} / s_{i} ; q\right)_{\lambda_{k-i}^{(i)}-\lambda_{k-i+1}^{(i)}}}\right| \leq C_{2}^{\lambda_{k-i}^{(i)}-\lambda_{k-i+1}^{(i)}} \quad(1 \leq i \leq N, i \leq j<k<\infty)
\end{aligned}
$$

for all $N$-partitions $\boldsymbol{\lambda}=\left(\lambda^{(1)}, \ldots, \lambda^{(N)}\right) \in \mathrm{P}^{N}$, with $C_{1}$ and $C_{2}$ in (3.12). This and (3.9) imply the estimate in (3.15) which, by the computation in (3.15), is equivalent to the second estimate in (3.13).

We observe all estimates in (B.1)-(B.2) are of the form

$$
\left|\frac{\left(q^{l} a s_{i} / s_{j} ; q\right)_{\theta}}{\left(q^{l} s_{i} / s_{j} ; q\right)_{\theta}}\right| \leq C^{\theta}
$$

where

$$
l=\lambda_{k-i+1}^{(i)}-\lambda_{k-j+1}^{(j)}+1, \quad a=t / q, \quad \theta=\lambda_{k-i}^{(i)}-\lambda_{k-i+1}^{(i)}, \quad C=C_{1}
$$

in (B.1) and

$$
l=-\lambda_{k-j}^{(j)}+\lambda_{k-i+1}^{(i)}, \quad a=q / t, \quad \theta=\lambda_{k-i}^{(i)}-\lambda_{k-i+1}^{(i)}, \quad C=C_{2}
$$

in (B.2). We prove (B.1)-(B.2) using three different kinds of estimates:

Lemma B.1. Let $\theta \in \mathbb{Z}_{\geq 0}, a \in \mathbb{C}, q, \kappa \in \mathbb{R}$ with either $|q|<1$ and $|\kappa|>1$ or $|q|>1$ and $|\kappa|<1$. Then the following estimates hold true,

(a) for all $l \in \mathbb{Z}$ and $u \in \mathbb{C} \backslash\{\mathbb{R}\}$ :

$$
\left|\frac{\left(q^{l} a u ; q\right)_{\theta}}{\left(q^{l} u ; q\right)_{\theta}}\right| \leq\left(1+\frac{|1-a|}{|\sin \arg (u)|}\right)^{\theta},
$$

(b) for all $m \in \mathbb{Z}_{\geq 0}, \ell \in \mathbb{Z}_{\geq 1}$ :

$$
\left|\frac{\left(q^{-\theta-m+1} a \kappa^{\ell}\right)_{\theta}}{\left(q^{-\theta-m+1} \kappa^{\ell}\right)_{\theta}}\right| \leq\left(1+|1-a| \frac{|\kappa|}{|1-| \kappa||}\right)^{\theta},
$$

(c) for all $m \in \mathbb{Z}_{\geq 0}, \ell \in \mathbb{Z}_{\geq 0}$ :

$$
\left|\frac{\left(q^{-\theta-m} a \kappa^{\ell}\right)_{\theta}}{\left(q^{-\theta-m} \kappa^{\ell}\right)_{\theta}}\right| \leq\left(1+|1-a| \frac{1}{|1-| q||}\right)^{\theta} .
$$


(The proof is given in Appendix B.2.)

Case A: For $j-i \notin N \mathbb{Z}_{\geq 0}$, we can use the estimate in (B.5): Since $s_{j+N}=\kappa s_{j}$ and $\kappa$ is real, we have $\left|\sin \arg \left(s_{j} / s_{i}\right)\right|=\left|\sin \arg \left(s_{j+N} / s_{i}\right)\right|=\left|\sin \arg \left(s_{i} / s_{j}\right)\right|$ for all $j \geq i$; since $\left|\sin \arg \left(s_{j} / s_{i}\right)\right| \geq \sigma$ for all $1 \leq i<j \leq N$ by assumption, $\left|\sin \arg \left(s_{j} / s_{i}\right)\right| \geq \sigma$ for all $1 \leq i \leq N$ and $j \geq i$ such that $j-i \neq N \mathbb{Z}_{\geq 0}$, and we get

$$
\left|\frac{\left(q^{l} a s_{i} / s_{j} ; q\right)_{\theta}}{\left(q^{l} s_{i} / s_{j} ; q\right)_{\theta}}\right| \leq\left(1+\frac{|1-a|}{\sigma}\right)^{\theta} \quad\left(j-i \notin N \mathbb{Z}_{\geq 0}\right)
$$

for all cases in (B.3)-(B.4). This proves that the estimates in (B.1)-(B.2) for all

$$
C_{1} \geq 1+\frac{|1-t / q|}{\sigma}, \quad C_{2} \geq 1+\frac{|1-q / t|}{\sigma}
$$

and for all cases $j-i \notin N \mathbb{Z}_{\geq 0}$.

We consider the remaining cases for (B.1) and (B.2) below in Cases B and C, respectively.

Case B: For $j-i \in N \mathbb{Z}_{\geq 1}$, we have $s_{j} / s_{i}=s_{i+\ell N} / s_{i}=\kappa^{\ell}$ for some $\ell \in \mathbb{Z}_{\geq 1}$, and we can use the estimate in (B.6):

$$
\left|\frac{\left(q^{-\theta-m+1} a s_{i} / s_{j} ; q\right)_{\theta}}{\left(q^{-\theta-m+1} s_{i} / s_{j} ; q\right)_{\theta}}\right| \leq\left(1+|1-a| \frac{|\kappa|}{|| \kappa|-1|}\right)^{\theta} \quad\left(j-i \in N \mathbb{Z}_{\geq 1}, m \in \mathbb{Z}_{\geq 0}\right) .
$$

We check that all cases in (B.1) for $j-i \in N \mathbb{Z}_{\geq 1}$ are covered by this: all $l$ in (B.3) for $j=i+\ell N$ can be written as (recall that $\lambda_{k}^{(i+\ell N)}=\lambda_{k}^{(i)}$ )

$$
l=-\left[\lambda_{k-i}^{(i)}-\lambda_{k-i+1}^{(i)}\right]-\left[\lambda_{k-i-\ell N+1}^{(i)}-\lambda_{k-i}^{(i)}\right]+1=-\theta-m+1
$$

with $m=\lambda_{k-i-\ell N+1}^{(i)}-\lambda_{k-i}^{(i)} \geq 0$ since $\ell \geq 1$ and $\lambda^{(i)}=\left(\lambda_{1}^{(i)}, \lambda_{2}^{(i)}, \ldots\right)$ is a partition. This proves that (B.1) holds true if

$$
C_{1} \geq 1+|1-t / q| \frac{|\kappa|}{|1-| \kappa||}
$$

for all cases $j-i \in N \mathbb{Z}_{\geq 1}$.

Case C: For $j-i \in N \mathbb{Z}_{\geq 0}$, we have $s_{j} / s_{i}=s_{i+\ell N} / s_{i}=\kappa^{\ell}$ for some $\ell \in \mathbb{Z}_{\geq 0}$, and we can use the estimate in (B.7):

$$
\left|\frac{\left(q^{-\theta-m} a s_{i} / s_{j} ; q\right)_{\theta}}{\left(q^{-\theta-m} s_{i} / s_{j} ; q\right)_{\theta}}\right| \leq\left(1+|1-a| \frac{1}{|1-| q||}\right)^{\theta} \quad\left(j-i \in N \mathbb{Z}_{\geq 0}, m \in \mathbb{Z}_{\geq 0}\right) .
$$

We check that all cases in (B.2) for $j-i \in N \mathbb{Z}_{\geq 0}$ are covered by this: all $l$ in can be written as

$$
l=-\left[\lambda_{k-i}^{(i)}-\lambda_{k-i+1}^{(i)}\right]-\left[\lambda_{k-i-\ell N}^{(i)}-\lambda_{k-i}^{(i)}\right]=-\theta-m
$$

with $m=\lambda_{k-i-\ell N}^{(i)}-\lambda_{k-i}^{(i)} \geq 0$. This proves that (B.2) holds true if

$$
C_{2} \geq 1+|1-q / t| \frac{1}{|1-| q||}
$$

for all cases $j-i \in N \mathbb{Z}_{\geq 0}$.

We proved that (B.1)-(B.2) holds true for all cases provided the conditions in (B.8), (B.9) and (B.10) all hold true; this is the case if we choose $C_{1}$ and $C_{2}$ as in (3.12). 


\section{B.2 Proof of Lemma B.1}

\section{B.2.1 Proof of the estimate in (B.5)}

We have

$$
\begin{aligned}
\text { LHS } & =\left|\prod_{n=0}^{\theta-1} \frac{1-q^{l+n} a u}{1-q^{l+n} u}\right|=\prod_{n=0}^{\theta-1}\left|1+(1-a) \frac{q^{l+n} u}{1-q^{l+n} u}\right| \\
& \leq \prod_{n=0}^{\theta-1}\left(1+|1-a|\left|\frac{q^{l+n} u}{1-q^{l+n} u}\right|\right) \leq \prod_{n=0}^{\theta-1}\left(1+\frac{|1-a|}{|\sin \arg (u)|}\right)=\text { RHS }
\end{aligned}
$$

using the estimate in (3.14) and $\left|\sin \arg \left(q^{l+n} u\right)\right|=|\sin \arg (u)|$ since $q$ is real.

\section{B.2.2 Proof of the estimate in (B.6)}

We have

$$
\begin{aligned}
\operatorname{LHS} & =\left|\prod_{n=0}^{\theta-1} \frac{1-q^{n-\theta-m+1} a \kappa^{\ell}}{1-q^{n-\theta-m+1} \kappa^{\ell}}\right|=\prod_{n=0}^{\theta-1}\left|1+(1-a) \frac{q^{-n-m} \kappa^{\ell}}{1-q^{-n-m} \kappa^{\ell}}\right| \\
& \leq \prod_{n=0}^{\theta-1}\left(1+|1-a|\left|\frac{q^{-n-m} \kappa^{\ell}}{1-q^{-n-m} \kappa^{\ell}}\right|\right) \leq \prod_{n=0}^{\theta-1}\left(1+|1-a| \frac{|\kappa|}{|1-| \kappa||}\right)=\mathrm{RHS},
\end{aligned}
$$

using

$$
\left|\frac{q^{-l} \kappa^{\ell}}{1-q^{-l} \kappa^{\ell}}\right| \leq \frac{|\kappa|}{|1-| \kappa||} \quad(l \geq 0, \ell \geq 1)
$$

the latter follows for the case $|\kappa|<1$ and $|q|>1$ from the following inequality: $x /(1-x)<$ $y /(1-x)$ for $0 \leq x<y<1$, and for the case $|\kappa|>1$ and $|q|<1$ :

$$
\left|\frac{q^{-l} \kappa^{\ell}}{1-q^{-l} \kappa^{\ell}}\right|=\left|\frac{1}{1-q^{l} \kappa^{-\ell}}\right| \leq \frac{1}{1-|1 / \kappa|}=\frac{|\kappa|}{|1-| \kappa||}
$$

since $1 /(1-x)<1 /(1-y)$ for $0 \leq x<y<1$.

\section{B.2.3 Proof of the estimate in (B.7)}

We have

$$
\begin{aligned}
\text { LHS } & =\left|\prod_{n=0}^{\theta-1} \frac{1-q^{n-\theta-m} a \kappa^{\ell}}{1-q^{n-\theta-m+1} \kappa^{\ell}}\right|=\prod_{n=1}^{\theta}\left|1+(1-a) \frac{q^{-n-m} \kappa^{\ell}}{1-q^{-n-m} \kappa^{\ell}}\right| \\
& \leq \prod_{n=1}^{\theta}\left(1+|1-a|\left|\frac{q^{-n-m} \kappa^{\ell}}{1-q^{-n-m} \kappa^{\ell}}\right|\right) \leq \prod_{n=1}^{\theta}\left(1+|1-a| \frac{1}{|1-| q||}\right)=\text { RHS },
\end{aligned}
$$

using

$$
\left|\frac{q^{-l} \kappa^{\ell}}{1-q^{-l} \kappa^{\ell}}\right| \leq \frac{\left|q^{-1}\right|}{|| q^{-1}|-1|}=\frac{1}{|1-| q||} \quad(l \geq 1, \ell \geq 0),
$$

as in the proof of (B.6). 


\section{Proof of Proposition 4.2}

We prove Proposition 4.2 using Lemma 4.3 in the main text.

\section{C.1 Proof of commutativity}

We prove (4.3). We note that the action of the Macdonald-Ruijsenaars operators in (2.1) on functions $x^{\lambda} f(x)$ can be written as [15]

$$
D_{N}^{ \pm}(x \mid q, t) x^{\lambda} f(x)=x^{\lambda} E_{N}^{ \pm}(x|s| q, t) f(x), \quad s_{i}=t^{N-i} q^{\lambda_{i}}
$$

with the modified Macdonald-Ruijsenaars operators

$$
\begin{aligned}
& E_{N}^{ \pm}(x|s| q, t)=\sum_{i=1}^{N} A_{N, i}\left(x \mid t^{ \pm}\right) s_{i}^{ \pm 1} T_{q, x_{i}}^{ \pm 1}, \\
& A_{N, i}\left(x \mid t^{ \pm 1}\right)=\prod_{j=1}^{i-1} \frac{1-t^{ \pm 1} x_{i} / x_{j}}{1-x_{i} / x_{j}} \prod_{k=i+1}^{N} \frac{1-t^{\mp 1} x_{k} / x_{i}}{1-x_{k} / x_{i}}
\end{aligned}
$$

(this can be proved by simple computations which we skip).

We also need properties of the function $\chi_{N}(x|y| q, t)$ in (4.7) which we summarize as follows.

Lemma C.1. The function $\chi_{N}(x|y| q, t)$ satisfies the following duality relation,

$$
\chi_{N}(x|y| q, t)=\chi_{N}(y|x| q, t / q) .
$$

Moreover,

$$
\begin{aligned}
& E_{N}^{ \pm}(x|y| q, t) \chi_{N}(x|y| q, t)=e_{1}\left(y^{ \pm 1}\right) \chi_{N}(x|y| q, t), \\
& E_{N}^{ \pm}(y|x| q, q / t) \chi_{N}(x|y| q, t)=e_{1}\left(x^{ \pm 1}\right) \chi_{N}(x|y| q, t)
\end{aligned}
$$

with

$$
e_{1}\left(x^{ \pm 1}\right)=x_{1}^{ \pm 1}+\cdots+x_{N}^{ \pm 1} .
$$

Proof. The definitions in (2.6) and (4.7) imply

$$
\chi_{N}(x|y| q, t)=\prod_{1 \leq i<j \leq N} \frac{\left(q x_{j} / x_{i} ; q\right)_{\infty}}{\left(q x_{j} / t x_{i} ; q\right)_{\infty}} \frac{\left(q y_{j} / y_{i} ; q\right)_{\infty}}{\left(t y_{j} / y_{i} ; q\right)_{\infty}} \varphi_{N}(x|y| q, t) .
$$

The product on the right-hand side is manifestly invariant under the transformation $(x, y, t) \mapsto$ $(y, x, q / t)$; the function $\varphi_{N}(x|y| q, t)$ has this invariance by (2.7). This proves (C.4).

The first identity in (C.5) is implied by $E_{N}^{ \pm}(x|s| q, t) f_{N}(x|s| q, t)=e_{1}\left(s^{ \pm 1}\right) f_{N}(x|s| q, t)$ proved in [15]; the second follows from the first and the duality in (C.4).

Equation (C.1) and Lemma 4.3 imply that the result we want to prove:

$$
D_{N}^{ \pm}(x \mid q, t) \mathcal{T}_{N}(x \mid q, t) x^{\lambda} f(x)=\mathcal{T}_{N}(x \mid q, t) D_{N}^{ \pm}(x \mid q, t) x^{\lambda} f(x),
$$

is equivalent to

$$
\left[E_{N}^{ \pm}(x|s| q, t) \prod_{i=1}^{N} \vartheta_{3}\left(s_{i} x_{i} / y_{i} \mid q\right) \chi_{N}(x|y| q, t) \prod_{1 \leq i<j \leq N}\left(1-y_{j} / y_{i}\right) f(y)\right]_{1, y}
$$




$$
=\left[\prod_{i=1}^{N} \vartheta_{3}\left(s_{i} x_{i} / y_{i} \mid q\right) \chi_{N}(x|y| q, t) \prod_{1 \leq i<j \leq N}\left(1-y_{j} / y_{i}\right)\left(E_{N}^{ \pm}(y|s| q, t) f(y)\right)\right]_{1, y} .
$$

The latter is obviously implied by the following two identities: first,

$$
\begin{aligned}
& E_{N}^{ \pm}(x|s| q, t) \prod_{i=1}^{N} \vartheta_{3}\left(s_{i} x_{i} / y_{i} \mid q\right) \chi_{N}(x|y| q, t) \\
& \quad=E_{N}^{\mp}\left(y\left|s^{-1}\right| q, q / t\right) \prod_{i=1}^{N} \vartheta_{3}\left(s_{i} x_{i} / y_{i} \mid q\right) \chi_{N}(x|y| q, t),
\end{aligned}
$$

and second,

$$
\begin{gathered}
{\left[\left(E_{N}^{\mp}\left(y\left|s^{-1}\right| q, q / t\right) \prod_{i=1}^{N} \vartheta_{3}\left(s_{i} x_{i} / y_{i} \mid q\right) \chi_{N}(x|y| q, t)\right) \prod_{1 \leq i<j \leq N}\left(1-y_{j} / y_{i}\right) f(y)\right]_{1, y}} \\
=\left[\prod_{i=1}^{N} \vartheta_{3}\left(s_{i} x_{i} / y_{i} \mid q\right) \chi_{N}(x|y| q, t) \prod_{1 \leq i<j \leq N}\left(1-y_{j} / y_{i}\right)\left(E_{N}^{ \pm}(y|s| q, t) f(y)\right)\right]_{1, y} .
\end{gathered}
$$

We first prove (C.7) in three steps, using the shorthand notation in (C.6). We start with

$$
E_{N}^{ \pm}(x|s| q, t) \prod_{i=1}^{N} \vartheta_{3}\left(s_{i} x_{i} / y_{i} \mid q\right)=\frac{q^{-1 / 2}}{1-q^{-1}} \prod_{i=1}^{N} \vartheta_{3}\left(s_{i} x_{i} / y_{i} \mid q\right)\left[e_{1}\left(x^{\mp 1}\right), E_{N}^{ \pm}(x|y| q, t)\right]
$$

proved by the following computation (we insert definitions and change the summation variable $n_{i} \pm 1 \rightarrow n_{i}$ in the third equality),

$$
\begin{aligned}
\mathrm{LHS} & =\sum_{i=1}^{N} A_{N, i}\left(x \mid t^{ \pm 1}\right) s_{i}^{ \pm 1} T_{q, x_{i}}^{ \pm 1} \sum_{n \in \mathbb{Z}^{N}} \prod_{j=1}^{N}\left(\frac{s_{j} x_{j}}{y_{j}}\right)^{n_{j}} q^{\frac{1}{2} n_{j}^{2}} \\
& =\sum_{n \in \mathbb{Z}^{N}} \sum_{i=1}^{N} A_{N, i}\left(x \mid t^{ \pm 1}\right)\left(\frac{y_{i}}{x_{i}}\right)^{ \pm 1}\left(\frac{s_{i} x_{i}}{y_{i}}\right)^{n_{i} \pm 1} q^{\frac{1}{2} n_{i}^{2} \pm n_{i}} \prod_{\substack{j=1 \\
j \neq i}}^{N}\left(\frac{s_{j} x_{j}}{y_{j}}\right)^{n_{j}} q^{\frac{1}{2} n_{j}^{2}} T_{q, x_{i}}^{ \pm 1} \\
& =\prod_{i=1}^{N} \vartheta_{3}\left(s_{i} x_{i} / y_{i} \mid q\right) \sum_{i=1}^{N} A_{N, i}\left(x \mid t^{ \pm 1}\right)\left(\frac{y_{i}}{x_{i}}\right)^{ \pm 1} q^{-1 / 2} T_{q, x_{i}}^{ \pm 1}=\mathrm{RHS}
\end{aligned}
$$

since

$$
\begin{aligned}
& {\left[e_{1}\left(x^{\mp 1}\right), E_{N}^{ \pm}(x|y| q, t)\right]} \\
& \quad=\sum_{i=1}^{N} A_{N, i}\left(x \mid t^{ \pm 1}\right) y_{i}^{ \pm 1}\left[x_{i}^{\mp 1}, T_{q, x_{i}}^{ \pm 1}\right]=\left(1-q^{-1}\right) \sum_{i=1}^{N} A_{N, i}\left(x \mid t^{ \pm 1}\right)\left(\frac{y_{i}}{x_{i}}\right)^{ \pm 1} T_{q, x_{i}}^{ \pm 1} .
\end{aligned}
$$

Next,

$$
\left[e_{1}\left(x^{\mp 1}\right), E_{N}^{ \pm}(x|y| q, t)\right] \chi_{N}(x|y| q, t)=\left[e_{1}\left(y^{ \pm 1}\right), E_{N}^{\mp}(y|x| q, q / t)\right] \chi_{N}(x|y| q, t),
$$

which is proved by

$$
\begin{aligned}
\mathrm{LHS} & =\left(e_{1}\left(x^{\mp 1}\right) e_{1}\left(y^{ \pm 1}\right)-E_{N}^{ \pm}(x|y| q, t) E_{N}^{\mp}(y|x| q, q / t)\right) \chi_{N}(x|y| q, t) \\
& =\left(e_{1}\left(y^{ \pm 1}\right) e_{1}\left(x^{\mp 1}\right)-E_{N}^{\mp}(y|x| q, q / t) E_{N}^{ \pm}(x|y| q, t)\right) \chi_{N}(x|y| q, t)=\mathrm{RHS}
\end{aligned}
$$


using (C.5) and

$$
\left[E_{N}^{ \pm}(x|y| q, t), E_{N}^{\mp}(y|x| q, q / t)\right]=0 ;
$$

the latter is verified by a simple computation using the definition in (C.2)-(C.3). Third,

$$
\begin{aligned}
& E_{N}^{\mp}\left(y\left|s^{-1}\right| q, q / t\right) \prod_{i=1}^{N} \vartheta_{3}\left(s_{i} x_{i} / y_{i} \mid q\right) \\
& \quad=\frac{q^{-1 / 2}}{1-q^{-1}} \prod_{i=1}^{N} \vartheta_{3}\left(s_{i} x_{i} / y_{i} \mid q\right)\left[e_{1}\left(y^{ \pm 1}\right), E_{N}^{\mp}(y|x| q, q / t)\right],
\end{aligned}
$$

which is proved similarly as (C.9):

$$
\begin{aligned}
\text { LHS } & =\sum_{i=1}^{N} A_{N, i}\left(y \mid(q / t)^{\mp 1}\right) s_{i}^{ \pm 1} T_{q, y_{i}}^{\mp 1} \sum_{n \in \mathbb{Z}^{N}} \prod_{j=1}^{N}\left(\frac{s_{j} x_{j}}{y_{j}}\right)^{n_{j}} q^{\frac{1}{2} n_{j}^{2}} \\
& =\sum_{n \in \mathbb{Z}^{N}} \sum_{i=1}^{N} A_{N, i}\left(y \mid(q / t)^{\mp 1}\right)\left(\frac{y_{i}}{x_{i}}\right)^{ \pm 1}\left(\frac{s_{i} x_{i}}{y_{i}}\right)^{n_{i} \pm 1} q^{\frac{1}{2} n_{i}^{2} \pm n_{i}} \prod_{\substack{j=1 \\
j \neq i}}^{N}\left(\frac{s_{j} x_{j}}{y_{j}}\right)^{n_{j}} q^{\frac{1}{2} n_{j}^{2}} T_{q, x_{i}}^{\mp 1} \\
& =\prod_{i=1}^{N} \vartheta_{3}\left(s_{i} x_{i} / y_{i} \mid q\right) \sum_{i=1}^{N} A_{N, i}\left(y \mid(q / t)^{\mp 1}\right)\left(\frac{y_{i}}{x_{i}}\right)^{ \pm 1} q^{-1 / 2} T_{q, y_{i}}^{\mp 1}=\mathrm{RHS}
\end{aligned}
$$

since

$$
\begin{aligned}
& {\left[e_{1}\left(y^{ \pm 1}\right), E_{N}^{\mp}(y|x| q, q / t)\right]} \\
& \quad=\sum_{i=1}^{N} A_{N, i}\left(y \mid(q / t)^{\mp 1}\right) x_{i}^{\mp 1}\left[y_{i}^{ \pm 1}, T_{q, y_{i}}^{\mp 1}\right]=\left(1-q^{-1}\right) \sum_{i=1}^{N} A_{N, i}\left(y \mid(q / t)^{\mp 1}\right)\left(\frac{y_{i}}{x_{i}}\right)^{ \pm 1} T_{q, y_{i}}^{\mp 1} .
\end{aligned}
$$

We are now ready to prove (C.7): we insert (C.9) into the LHS in (C.7), use (C.10) and (C.11), and obtain the RHS in (C.7).

To conclude our proof of (4.3), we prove (C.8) by the following computation, using the definitions in (C.2)-(C.3) and the basic property $\left[\left(T_{q, y_{i}}^{\mp 1} g_{1}(y)\right) g_{2}(y)\right]_{1, y}=\left[g_{1}(y)\left(T_{q, y_{i}}^{ \pm 1} g_{2}(y)\right)\right]_{1, y}$ of the constant term:

$$
\begin{aligned}
\mathrm{LHS} & =\left[\sum_{i=1}^{N} A_{N, i}\left(y \mid(q / t)^{\mp 1}\right) s_{i}^{ \pm 1}\left(T_{q, y_{i}}^{\mp 1} \prod_{i=1}^{N} \vartheta_{3}\left(s_{i} x_{i} / y_{i} \mid q\right) \chi_{N}(y|x| q, t)\right)_{1 \leq i<j \leq N}\left(1-y_{j} / y_{i}\right) f(y)\right]_{1, y} \\
& =\left[\prod_{i=1}^{N} \vartheta_{3}\left(s_{i} x_{i} / y_{i} \mid q\right) \chi_{N}(y|x| q, t) \sum_{i=1}^{N}\left(T_{q, y_{i}}^{ \pm 1} A_{N, i}\left(y \mid(q / t)^{\mp 1}\right) s_{i}^{ \pm 1} \prod_{1 \leq i<j \leq N}\left(1-y_{j} / y_{i}\right) f(y)\right)\right]_{1, y} \\
& =\operatorname{RHS}
\end{aligned}
$$

provided

$$
\frac{T_{q, y_{i}}^{ \pm 1} A_{N, i}\left(y \mid(q / t)^{\mp 1}\right) \prod_{1 \leq i<j \leq N}\left(1-y_{j} / y_{i}\right)}{\prod_{1 \leq i<j \leq N}\left(1-y_{j} / y_{i}\right)}=A_{N, i}\left(y \mid t^{ \pm}\right) ;
$$

the latter holds true for the coefficients $A_{N, i}(x \mid t)$ in (C.3), as is easily verified:

$$
\text { LHS }=\prod_{j=1}^{i-1} \frac{1-(q / t)^{\mp 1} q^{ \pm 1} y_{i} / y_{j}}{1-q^{ \pm 1} y_{i} / y_{j}} \prod_{k=i+1}^{N} \frac{1-(q / t)^{ \pm 1} y_{k} / q^{ \pm 1} y_{i}}{1-y_{k} / q^{ \pm 1} y_{i}} \prod_{j=1}^{i-1} \frac{1-q^{ \pm 1} y_{i} / y_{j}}{1-y_{i} / y_{j}}
$$




$$
\times \prod_{k=i+1}^{N} \frac{1-y_{k} / q^{ \pm 1} y_{i}}{1-y_{k} / y_{i}}=\mathrm{RHS}
$$

\section{C.2 Eigenfunction property}

The eigenfunctions $x^{\lambda} f_{N}(x|s| q, t)$ of $D_{N}^{ \pm}(x|s| q, t)$ are unique, and (4.3) therefore implies that $x^{\lambda} f_{N}(x|s| q, t)$ also are eigenfunction of $\mathcal{T}_{N}(x \mid q, t)$.

We are left to determine the eigenvalues. For that, we introduce some notation: the space of formal power series $\mathbb{C}\left[\left[x_{2} / x_{1}, \ldots, x_{N} / x_{N-1}\right]\right]$ is spanned by monomials

$$
\left(x_{2} / x_{1}\right)^{\alpha_{1}} \cdots\left(x_{N} / x_{N-1}\right)^{\alpha_{N_{1}}}
$$

with $\alpha=\left(\alpha_{1}, \ldots, \alpha_{N-1}\right) \in \mathbb{Z}_{\geq 0}^{N-1}$. Any such monomial can be written as $x^{\mu}=x_{1}^{\mu_{1}} \cdots x_{N}^{\mu_{N}}$ with $\mu=\mu(\alpha)$ given by

$$
\mu_{1}(\alpha)=-\alpha_{1}, \quad \mu_{i}(\alpha)=\alpha_{i-1}-\alpha_{i} \quad(i=2, \ldots, N-1), \quad \mu_{N}(\alpha)=\alpha_{N-1} .
$$

One can verify that the action of the operator in (4.2) is triangular on this basis in the following sense,

$$
\mathcal{T}_{N}(x \mid q, t) x^{\lambda} x^{\mu(\alpha)}=\varepsilon(\lambda+\mu(\alpha)) x^{\lambda}\left(x^{\mu(\alpha)}+\sum_{\beta>\alpha} v_{\alpha \beta} x^{\mu(\beta)}\right)
$$

for some coefficients $v_{\alpha \beta}$, where $\beta \geq \alpha$ means that $\beta_{i} \geq \alpha_{i}$ for all $i=1, \ldots, N-1$ (this follows from

$$
q^{\frac{1}{2} \Delta} x^{\lambda} x^{\mu}=\varepsilon(\lambda+\mu) x^{\lambda+\mu}, \quad \varepsilon(\lambda+\mu)=q^{\frac{1}{2} \sum_{i=1}^{N}\left(\lambda_{i}+\mu_{i}+(N-i) \beta\right)^{2}}
$$

used already in the main text, and the fact that all functions of $x$ appearing in the definition of $\mathcal{T}_{N}(x \mid q, t)$ in $(4.2)$ can be expanded as power series in $\left.\mathbb{C}\left[\left[x_{2} / x_{1}, \ldots, x_{N} / x_{N-1}\right]\right]\right)$. Since

$$
x^{\lambda} f_{N}(x|s| q, t)=x^{\lambda}\left(1+\sum_{\alpha>0} b_{\alpha} x^{\mu(\alpha)}\right)
$$

for some coefficients $b_{\alpha}$, the eigenvalue is $\varepsilon(\lambda)=\varepsilon(s \mid q)$ in (4.5).

\section{Identities}

For clarify, and for the convenience of the reader, we state and prove two identities used in the main text.

First, the identity

$$
\sum_{\lambda \in \mathrm{P}} \alpha^{|\lambda|}=\frac{1}{(\alpha ; \alpha)_{\infty}} \quad(|\alpha|<1),
$$

which goes back to Euler, is important in our proof of Theorem 3.7. It is proved by the following elementary computation making absolute convergence of the series manifest,

$$
\begin{aligned}
\text { LHS } & =\lim _{M \rightarrow \infty} \sum_{\lambda_{1} \geq \lambda_{2} \geq \cdots \geq \lambda_{M} \geq 0} \alpha^{\lambda_{1}+\lambda_{2}+\cdots+\lambda_{M}} \\
& =\lim _{M \rightarrow \infty} \sum_{\lambda_{1}=\lambda_{2}}^{\infty} \sum_{\lambda_{2}=\lambda_{3}}^{\infty} \ldots \sum_{\lambda_{M-1}=\lambda_{M}}^{\infty} \sum_{\lambda_{M}=0}^{\infty} \alpha^{\lambda_{1}+\lambda_{2}+\cdots+\lambda_{M}}
\end{aligned}
$$




$$
\begin{aligned}
& =\lim _{M \rightarrow \infty} \sum_{\lambda_{2}=\lambda_{3}}^{\infty} \cdots \sum_{\lambda_{M-1}=\lambda_{M}}^{\infty} \sum_{\lambda_{M}=0}^{\infty} \frac{1}{1-\alpha} \alpha^{2 \lambda_{2}+\lambda_{3}+\cdots+\lambda_{M}} \\
& =\lim _{M \rightarrow \infty} \sum_{\lambda_{3}=\lambda_{4}}^{\infty} \cdots \sum_{\lambda_{M-1}=\lambda_{M}}^{\infty} \sum_{\lambda_{M}=0}^{\infty} \frac{1}{(1-\alpha)\left(1-\alpha^{2}\right)} \alpha^{3 \lambda_{3}+\lambda_{4}+\cdots+\lambda_{M}}=\cdots \\
& =\lim _{M \rightarrow \infty} \frac{1}{(1-\alpha)} \frac{1}{\left(1-\alpha^{2}\right)} \cdots \frac{1}{\left(1-\alpha^{M}\right)}=\mathrm{RHS},
\end{aligned}
$$

summing repeatedly the geometric series.

Second, we state and prove an identity used in the proof of Fact 3.6 and Remark 4.6.

Lemma D.1. For $p \in \mathbb{C}$, let $x_{i} \in \mathbb{C}$ be given for $i=1, \ldots, N$, and extend this definition to all $i \geq 1$ by $x_{i+N}=p x_{i}$. Then

$$
\prod_{i=1}^{N} \prod_{j>i} \frac{\left(a x_{j} / x_{i} ; q\right)_{\infty}}{\left(b x_{j} / x_{i} ; q\right)_{\infty}}=\prod_{1 \leq i<j \leq N} \frac{\left(a x_{j} / x_{i} ; q, p\right)_{\infty}}{\left(b x_{j} / x_{i} ; q, p\right)_{\infty}} \prod_{1 \leq i \leq j \leq N} \frac{\left(p a x_{i} / x_{j} ; q, p\right)_{\infty}}{\left(p b x_{i} / x_{j} ; q, p\right)_{\infty}}
$$

for all $a, b, q \in \mathbb{C}$.

Proof. We note that $(z ; q, p)=\prod_{m=0}^{\infty}\left(p^{m} x ; q\right)_{\infty}$, and thus

$$
\begin{aligned}
\text { LHS } & =\prod_{1 \leq i<j \leq N} \prod_{m=0}^{\infty} \frac{\left(a x_{j+m N} / x_{i} ; q\right)_{\infty}}{\left(b x_{j+m N} / x_{i} ; q\right)_{\infty}} \prod_{1 \leq j \leq i \leq N} \prod_{m=1}^{\infty} \frac{\left(a x_{j+m N} / x_{i} ; q\right)_{\infty}}{\left(b x_{j+m N} / x_{i} ; q\right)_{\infty}} \\
& =\prod_{1 \leq i<j \leq N} \prod_{m=0}^{\infty} \frac{\left(p^{m} a x_{j} / x_{i} ; q\right)_{\infty}}{\left(p^{m} b x_{j} / x_{i} ; q\right)_{\infty}} \prod_{1 \leq j \leq i \leq N} \prod_{m=1}^{\infty} \frac{\left(p^{m} a x_{j} / x_{i} ; q\right)_{\infty}}{\left(p^{m} b x_{j} / x_{i} ; q\right)_{\infty}}=\mathrm{RHS},
\end{aligned}
$$

inserting $x_{j+m N}=p^{m} x_{j}$.

\section{Acknowledgements}

We would like to thank F. Atai, A. Negut, and V. Pasquier for useful discussions. We thank J. Lamers for a helpful comment on the manuscript. We are grateful to careful referees for remarks helping us to improve our paper. This work is supported by VR Grant No 201605167 (E.L.) and by JSPS Kakenhi Grants (B) 15 H03626 (M.N.), (C) 19 K03512 (J.S.). MN gratefully acknowledges financial support by the Knut and Alice Wallenberg foundation (KAW 2019.0525). We are grateful to the Stiftelse Olle Engkvist Byggmästare, Contract 184-0573, for financial support.

\section{References}

[1] Atai F., Langmann E., Series solutions of the non-stationary Heun equation, SIGMA 14 (2018), 011, 32 pages, arXiv:1609.02525.

[2] Atai F., Langmann E., Exact solutions by integrals of the non-stationary elliptic Calogero-Sutherland equation, J. Integrable Syst. 5 (2020), xyaa001, 26 pages, arXiv:1908.00529.

[3] Awata H., Kanno H., Mironov A., Morozov A., On a complete solution of the quantum Dell system, J. High Energy Phys. 2020 (2020), no. 4, 212, 30 pages, arXiv:1912.12897.

[4] Braden H.W., Marshakov A., Mironov A., Morozov A., On double-elliptic integrable systems. I. A duality argument for the case of SU(2), Nuclear Phys. B 573 (2000), 553-572, arXiv:hep-th/9906240.

[5] Felder G., Varchenko A., The q-deformed Knizhnik-Zamolodchikov-Bernard heat equation, Comm. Math. Phys. 221 (2001), 549-571, arXiv:math.QA/9809139. 
[6] Felder G., Varchenko A., $q$-deformed KZB heat equation: completeness, modular properties and $\mathrm{SL}(3, \mathbb{Z})$, Adv. Math. 171 (2002), 228-275, arXiv:math.QA/0110081.

[7] Felder G., Varchenko A., Hypergeometric theta functions and elliptic Macdonald polynomials, Int. Math. Res. Not. 2004 (2004), 1037-1055, arXiv:math.QA/0309452.

[8] Fock V., Gorsky A., Nekrasov N., Rubtsov V., Duality in integrable systems and gauge theories, J. High Energy Phys. 2000 (2000), no. 7, 028, 40 pages, arXiv:hep-th/9906235.

[9] Fukuda M., Ohkubo Y., Shiraishi J., Non-stationary Ruijsenaars functions for $\kappa=t^{-1 / N}$ and intertwining operators of Ding-Iohara-Miki algebra, arXiv:2002.00243.

[10] Hallnäs M., Ruijsenaars S., Joint eigenfunctions for the relativistic Calogero-Moser Hamiltonians of hyperbolic type. III. Factorized asymptotics, arXiv:1905.12918.

[11] Koroteev P., Shakirov S., The quantum DELL system, Lett. Math. Phys. 110 (2020), 969-999, arXiv:1906.10354.

[12] Langmann E., Explicit solution of the (quantum) elliptic Calogero-Sutherland model, Ann. Henri Poincaré 15 (2014), 755-791, arXiv:math-ph/0401029.

[13] Macdonald I.G., Symmetric functions and Hall polynomials, 2nd ed., Oxford Mathematical Monographs, The Clarendon Press, Oxford University Press, New York, 1995.

[14] Nekrasov N.A., Seiberg-Witten prepotential from instanton counting, Adv. Theor. Math. Phys. 7 (2003), 831-864, arXiv:hep-th/0206161.

[15] Noumi M., Shiraishi J., A direct approach to the bispectral problem for the Ruijsenaars-Macdonald $q$ difference operators, arXiv:1206.5364.

[16] Olshanetsky M.A., Perelomov A.M., Quantum integrable systems related to Lie algebras, Phys. Rep. 94 (1983), 313-404.

[17] Ruijsenaars S.N.M., Complete integrability of relativistic Calogero-Moser systems and elliptic function identities, Comm. Math. Phys. 110 (1987), 191-213.

[18] Ruijsenaars S.N.M., Hilbert-Schmidt operators vs. integrable systems of elliptic Calogero-Moser type. I. The eigenfunction identities, Comm. Math. Phys. 286 (2009), 629-657.

[19] Ruijsenaars S.N.M., Hilbert-Schmidt operators vs. integrable systems of elliptic Calogero-Moser type. II. The $A_{N-1}$ case: first steps, Comm. Math. Phys. 286 (2009), 659-680.

[20] Shiraishi J., A conjecture about raising operators for Macdonald polynomials, Lett. Math. Phys. 73 (2005), 71-81, arXiv:math.QA/0503727.

[21] Shiraishi J., Affine screening operators, affine Laumon spaces and conjectures concerning non-stationary Ruijsenaars functions, J. Integrable Syst. 4 (2019), xyz010, 30 pages, arXiv:1903.07495. 